\title{
Design, Modeling, and Performance Analysis of Multi-Antenna Heterogeneous Cellular Networks
}

\author{
Arman Shojaeifard, Member, IEEE, Khairi Ashour Hamdi, Senior Member, IEEE, \\ Emad Alsusa, Senior Member, IEEE, Daniel K. C. So, Senior Member, IEEE, \\ Jie Tang, Member, IEEE, and Kai-Kit Wong, Fellow, IEEE
}

\begin{abstract}
This paper presents a stochastic geometry-based framework for the design and analysis of downlink multiuser multiple-input multiple-output (MIMO) heterogeneous cellular networks with linear zero-forcing transmit precoding and receive combining, assuming Rayleigh fading channels and perfect channel state information. The generalized tiers of base stations may differ in terms of their Poisson point process spatial density, number of transmit antennas, transmit power, artificial-biasing weight, and number of user equipments served per resource block. The spectral efficiency of a typical user equipped with multiple receive antennas is characterized using a non-direct moment-generating-function-based methodology with closed-form expressions of the useful received signal and aggregate network interference statistics systematically derived. In addition, the area spectral efficiency is formulated under different space-division multiple-access and single-user beamforming transmission schemes. We examine the impact of different cellular network deployments, propagation conditions, antenna configurations, and MIMO setups on the achievable performance through theoretical and simulation studies. Based on the stateof-the-art system parameters, the results highlight the inherent limitations of baseline single-input single-output transmission and conventional sparse macro-cell deployment, as well as the promising potential of multi-antenna communications and smallcell solution in interference-limited cellular environments.
\end{abstract}

Index Terms-Multi-antenna communications, downlink heterogeneous cellular networks, stochastic geometry theory.

\section{INTRODUCTION}

$\mathbf{T}$ HE RAPID deployment of cellular network infrastructure is considered a de facto solution for supporting the anticipated explosive traffic growth [1]. While legacy wireless communication systems are fast approaching the informationtheoretic capacity limits, network densification can elevate

Manuscript received July 10, 2015; revised December 4, 2015 and April 1, 2016; accepted May 14, 2016. Date of publication May 24, 2016; date of current version July 12, 2016. This work was supported by the Engineering and Physical Sciences Research Council (EPSRC) under grants EP/J021768/1 and EP/N008219/1. This paper was presented at the 2016 IEEE 83rd Vehicular Technology Conference (VTC2016-Spring), Nanjing, China, May 2016. The associate editor coordinating the review of this paper and approving it for publication was Z. Dawy.

A. Shojaeifard and K.-K. Wong are with the Department of Electronic and Electrical Engineering, University College London, London WC1E 7JE, U.K (e-mail: a.shojaeifard@ucl.ac.uk; kai-kit.wong@ucl.ac.uk).

K. A. Hamdi, E. Alsusa, and D. K. C. So are with the School of Electrical and Electronic Engineering, University of Manchester, Manchester M13 9PL, U.K. (e-mail: k.hamdi@manchester.ac.uk; e.alsusa@manchester.ac.uk; d.so@manchester.ac.uk).

J. Tang is with the School of Electronic and Information Engineering, South China University of Technology, Guangzhou 510641, China (e-mail: eejtang@scut.edu.cn).

Digital Object Identifier 10.1109/TCOMM.2016.2572187 transmission rates further through shortening the transmitterreceiver distance and serving fewer users in each cell [2]. This approach can be adopted via deploying different tiers of base stations (BSs), relay nodes, and distributed antennas known as heterogeneous cellular network (HetNet) [3], [4]. A different realization is cloud radio access network (CRAN) where nodes are remote radio units (RRUs) connected through the fronthaul to baseband units (BBUs) for processing [5]. Although these cellular network architectures face different technical challenges, with extreme densification, interference has become the main performance bottleneck in these systems.

Analyzing the interference behavior has become increasingly challenging with the large number of interfering sources significantly increasing the computational complexity of system-level simulations [6]. Furthermore, the rapid, irregular, heterogeneous, and overlapping placement of network nodes has rendered the use of conventional deterministic hexagonalgrid model obsolete [7], [8]. A promising alternative approach is to characterize and evaluate interference under random network topologies using tools from applied probability, in particular point processes and stochastic geometry theory [9]. This theoretical approach allows for the derivation of tractable and computationally-efficient spatially-averaged performance metrics which can greatly assist with cellular network design, deployment, and planning through depicting the fundamental performance trends, bounds, and trade-offs.

On the other hand, interference avoidance in existing macrocell deployments is typically enforced by allocating different parts of the spectrum to neighboring cells, which comes at the expense of poor spectrum exploitation [10]. In contrast, emerging cellular networks employ an aggressive frequency reuse strategy where different nodes can access the same system resources [11]. This trend highlights the importance of effective interference mitigation schemes towards facilitating efficient bandwidth utilization. Employing multiple antennas at the radio nodes is a prominent transmission technology for suppressing interference and improving network throughput by exploiting the spatial degrees of freedom [12].

\section{A. State-of-the-Art}

Interference management schemes based on multiple-input multiple-output (MIMO) communications have been extensively investigated in the literature [13], [14]. Considering perfect channel state information (CSI) is available at 
the transmitter, linear precoding techniques such as zeroforcing $(\mathrm{ZF})$ can be used to eliminate inter-antenna and multi-user interference and provide diversity gain. Linear $\mathrm{ZF}$ precoding fascilitates the same multiplexing gain as the high-complexity capacity-achieving dirty-paper-coding (DPC), whilst being significantly easier to implement in practice [15]. On the other hand, neglecting inter-cell interference may significantly limit performance particularly for the cell-edge users [16]. With multiple antennas at the receiver, however, linear beamforming techniques such as ZF combining can be applied to improve the intended signal strength or/and suppress interference [17], [18].

Most existing works on MIMO communications with random topologies however focus on ad hoc networks where the distance between a transmitter-receiver pair can in theory be longer than that from the receiver to the closest interferer [12], [19]. On the contrary, in cellular networks, each user equipment (UE) is typically associated with its closest BS of a certain tier which provides the greatest received signal power [20]. While there are well-established stochastic geometry-based frameworks for the design and analysis of randomly-deployed cellular networks with baseline singleinput single-output (SISO) transmission in the literature (see e.g., [6], [21], [22]), the extension to MIMO scenarios is an active area of research.

In [23], the performance of different space-division multiple-access (SDMA), single-user beamforming (SUBF), and baseline SISO transmission schemes in multiple-input single-output (MISO) HetNets were analyzed and compared using tools from stochastic orders. In particular, considering interference-limited cases, bounded closed-form expressions for the area spectral efficiency under these different transmission schemes were derived. The signal-to-interference ratio (SIR) distribution for generalized multi-user MIMO HetNets was derived using Toeplitz matrix representation in [24]. The interference statistics for multi-user MISO systems in a hybrid network topology, with a reference circular-shaped cell surrounded by a Poisson point process (PPP)-based interference field, was characterized in [25]. In particular, simplified expressions for the coverage probability and ergodic rate were provided through moment matching of the interference statistics with the Gamma distribution. On the other hand, with multiple antennas at the receiving user equipments (UEs), the performance of the SDMA transmission scheme in singletier open-loop MIMO cellular networks with linear ZF and minimum-mean-square-estimation (MMSE) receivers was investigated in [26]. The work in [27] utilized an alternative equivalent-in-distribution (EiD)-based approach for the performance analysis of single-tier cellular networks with different MIMO setups. With the aid of the Gil-Pelaez theorem, coverage and rate expressions for homogeneous network deployments with Gamma-distributed per-link power gains were derived in [28].

\section{B. Paper Contributions}

Motivated by the above, in this paper, a theoretical model for the design, modeling, and performance analysis of downlink
HetNets with multiple antennas at all nodes is provided. We employ linear ZF precoding at the transmitting BSs in order to cancel inter-user interference whilst making use of the remaining degrees of freedom for enhancing system throughput according to the numbers of available transmit antennas and UEs requesting service per resource block. At the receiver end, we utilize a linear $\mathrm{ZF}$ combiner for inter-cell interference cancellation, signal power boosting, or a combination of both depending on the number of available receive antennas. A systematic methodology for developing closedform expressions for the conditional statistics of the intended signal power and aggregate network interference over Rayleigh fading channels is accordingly provided. The proposed nondirect moment-generating-function (MGF)-based framework facilitates the exact calculation of the spectral efficiency whilst being more analytically tractable and computationally-efficient than the existing direct probability density function (pdf)based methods. The proposed theoretical model can serve as a benchmark tool for the performance evaluation of MIMO cellular networks comprising generalized tiers of BSs differing in terms of their transmission scheme, deployment density, number of transmit antennas, transmission power, artificialbias, and number of multi-antenna equipped UEs served in each resource block. The validity of the analytical framework is confirmed through extensive Monte-Carlo simulations for various settings of system parameters.

Several useful network design insights are obtained from our findings. We study the inherent trade-off between interference cancellation and signal power enhancement and show that the optimal number of receive antennas used towards mitigating inter-cell interference increases with greater deployment density, number of transmit/receive antennas, signal-tonoise ratios (SNRs), and path-loss exponents. Moreover, the user and area spectral efficiencies in multi-antenna homogeneous/heterogeneous cellular networks with different transmission schemes are examined. The user spectral efficiency is shown to be higher when serving a single user in each resource block as in the case of SUBF over SDMA where multiple users are simultaneously served. In turn, the area spectral efficiency of the latter scheme is shown to be greater, especially under higher number of transmit antennas, medium/high SNR regions, and minor distance-dependent path-loss. We observe that the improvement in performance from adding more transmit antennas is higher when the UEs are equipped with fewer receive antennas. On the other hand, having more receive antennas is shown to enhance performance; the gain however becomes smaller as the total number moves away from one receive antenna and when more transmit antennas are available. Another observation is that the spectral efficiency of a typical user in a macro-cell deployment can be greatly enhanced through deployment of small-cells. This performance gain can be further increased by appropriate offloading of traffic from macro-cells onto small-cells through artificial-bias. The results confirm the promising potential of multi-antenna HetNets with small-cell solution in achieving higher area spectral efficiencies over conventional homogeneous cellular networks with baseline SISO transmission. 


\section{Paper Organization}

The reminder of this paper is organized as follows. The modeling and analysis for MIMO homogeneous cellular networks is provided in Section II. In Section III, the proposed methodology is extended to generalized multi-antenna multitier cellular networks. In Section IV, extensive theoretical and simulation studies are conducted towards depicting network design guidelines. Finally, the paper is concluded in Section V.

Notation: A bold uppercase $\boldsymbol{X}$ denotes a matrix and a bold lowercase $\boldsymbol{x}$ denotes a vector; the superscripts $T$ and $\dagger$ respectively stand for the transpose and the Hermitian-transpose operations; $\mathbb{E}_{x}\{f(x)\}$ depicts the average of a function $f(x)$ with respect to a random variable $x ; \mathscr{P}(x)$ is the probability of an event $x$ occurring; $\mathcal{P}_{x}($.$) represents the pdf of a random$ variable $x ; \mathcal{M}_{x}(z)=\mathbb{E}_{x}\{\exp (-z x)\}$ is the MGF of a random variable $x ;|x|$ is the absolute value of $x ;\|x\|$ denotes the norm of a vector $\boldsymbol{x} ;\|\boldsymbol{X}\|$ denotes the Frobenius norm of a matrix $\boldsymbol{X} ; \Gamma(x)=\int_{0}^{+\infty} \exp (-s) s^{x-1} \mathrm{~d} s$ is the Gamma function; $\Gamma(y, x)=\int_{x}^{+\infty} \exp (-s) s^{y-1} \mathrm{~d} s$ is the (upper) incomplete Gamma function; ${ }_{2} F_{1}(a, b ; c ; d)=\sum_{x=0}^{+\infty} \frac{(a)_{x}(b)_{x}}{(c)_{x} x !} d^{x}$, where $(n)_{x}=n(n+1) \ldots(n+x-1)$, is the Gauss hypergeometric function; ${ }_{2} \tilde{F}_{1}(a, b ; c ; d)=\frac{2 F_{1}(a, b ; c ; d)}{\Gamma(c)}$ is the Regularized Gauss hypergeometric function.

\section{Homogeneous Cellular Networks}

In order to provide the reader with a clear understanding of the proposed theoretical framework, we first consider MIMO homogeneous cellular networks and subsequently extend the analysis to the generalized multi-antenna HetNet scenario. Specifically, consider the downlink of a cellular network comprising BSs distributed according to a stationary PPP $\Phi^{(b)}$ with spatial density $\lambda^{(b)}$. The PPP-based abstraction model is proved to be an accurate representation for the dense, irregular, overlapping, and heterogeneous deployment of nodes in emerging cellular networks [22]. By employing the Campbell-Mecke theorem [29], the analysis is carried out for an arbitrary user assumed to be located at the origin. Let $Y_{l}$ and $\left\|Y_{l}\right\|$ respectively represent the location on the twodimensional Euclidean plane $\mathbb{R}^{2}$ and the Euclidean distance of the $l$-th BS with respect to the reference UE. A co-channel deployment with universal frequency reuse allowing all cells to utilize the entire system bandwidth is taken into account.

We consider the case where the BSs and UEs are respectively equipped with $N^{t x}$ transmit and $N^{r x}$ receive antennas. The air interface technology under consideration is multiuser MIMO where each BS simultaneously serves multiple UEs $U$ in each resource block through independent spatial data streams with an equal per-user transmit power of $P$ [23]. Note that this assumption follows from the argument that the Poisson-Voronoi coverage regions of the randomly-deployed BSs with equivalent operational parameters are of a similar size with high probability. We assume ideal closed-loop MIMO communications where each transmitter has perfect knowledge of all of its users channels, and each receiver has perfect knowledge of its own channel and is further capable of perfectly estimating the channels from the interfering BSs. The perfect CSI at each transmitter is then used to design linear
ZF precoders for canceling the inter-user interference whilst utilizing any remaining degrees of freedom for enhancing the system throughput. In order to facilitate simultaneous service to all users under zero inter-user interference, the number of transmit antennas at each serving BS is assumed to be at least equal to its total number of users, i.e., $N^{t x} \geq U$. The linear $\mathrm{ZF}$ combiner at the user side is then invoked to perform intercell interference cancellation or/and signal power boosting depending on the number of receive antennas.

In this paper, we consider uncorrelated unit-mean Rayleigh fading for the intended and interfering links. The complex Gaussian channel vector between the $n$-th antenna of the $l$-th BS to the typical user with independent and identicallydistributed (i.i.d.) $\mathcal{C N}(0,1)$ entries is denoted by $\boldsymbol{g}_{Y_{l}, n} \in$ $\mathbb{C}^{N^{r x} \times 1}$. Hence, the link from the $n$-th antenna of the $l$-th BS to the reference UE considering unbounded distance-dependent path-loss with exponent $\beta(>2)$ is given by $\left\|Y_{l}\right\|^{-\frac{\beta}{2}} \boldsymbol{g}_{Y_{l}, n}$. The channel matrix from the $l$-th BS to the arbitrary user is denoted by $\boldsymbol{G}_{Y_{l}}=\left[\boldsymbol{g}_{Y_{l}, n}\right]_{1 \leq n \leq N^{t x}} \in \mathbb{C}^{N^{r x} \times N^{t x}}$. Note that all fading channels are assumed to be quasi-stationary and frequencyflat. In addition, $\boldsymbol{\eta} \in \mathbb{C}^{N^{r x} \times 1}$ is used to represent the circularlysymmetric additive white Gaussian noise (AWGN) vector with zero-mean and variance $\sigma^{2}$ per entry at the reference UE.

The cellular association strategy under consideration is concerned with connecting the reference UE to its closest deployed BS $l^{*}$. This can be mathematically formulated as

$$
Y_{l^{*}}=\arg \max \left(\left\|Y_{l}\right\|^{-\beta}\right), \quad \forall Y_{l} \in \Phi^{(b)} .
$$

Hence, the pdf of the random reference transmitter-receiver distance can be expressed as [9]

$$
\mathcal{P}_{\| Y_{l^{*} \|}}\left(r_{0}\right)=2 \pi r_{0} \lambda^{(b)} \exp \left(-\pi \lambda^{(b)} r_{0}^{2}\right) .
$$

In addition, the cumulative distribution function (cdf) of the statistical distance $\left\|Y_{L}\right\|=r_{L}$ between the reference user and the $L$-th closest interfering BS conditioned on the distance of the reference transmitter-receiver pair being $\left\|Y_{l^{*}}\right\|=r_{0}$ is equivalent to the probability of the event where there are at least $L$ sources in the region between the circles of radius $r_{0}$ and $r_{L}$. Hence, the corresponding conditional pdf can be written as [30]

$$
\begin{aligned}
\mathcal{P}_{\left\|Y_{L}\right\| \mid \| Y_{l^{*} \|}}\left(r_{L} \mid r_{0}\right)= & \frac{2 \pi r_{L} \lambda^{(b)}}{\Gamma(L)}\left(\pi\left(r_{L}^{2}-r_{0}^{2}\right) \lambda^{(b)}\right)^{L-1} \\
& \times \exp \left(-\pi\left(r_{L}^{2}-r_{0}^{2}\right) \lambda^{(b)}\right) .
\end{aligned}
$$

The baseband received signal vector $\boldsymbol{y} \in \mathbb{C}^{N^{r x} \times 1}$ at the reference UE in the multi-cell multi-user downlink MIMO cellular network can be mathematically described as

$$
\boldsymbol{y}=\sum_{Y_{l} \in \Phi^{(b)}}\left\|Y_{l}\right\|^{-\frac{\beta}{2}} \boldsymbol{G}_{Y_{l}} \sum_{k=1}^{U} \boldsymbol{x}_{Y_{l}, k}+\boldsymbol{\eta}
$$

where $\boldsymbol{x}_{Y_{l}, k} \in \mathbb{C}^{N^{t x} \times 1}$ denotes the transmit signal vector from the $l$-th BS intended for its $k$-th user subject to an equal peruser power constraint $\mathbb{E}\left\{\left\|\boldsymbol{x}_{Y_{l}, k}\right\|^{2}\right\}=P$, where $Y_{l} \in \Phi^{(b)}$ and $k \in \mathcal{U}=\{1,2, \ldots, U\}$. Considering linear precoding at the transmitter, we can further write

$$
\boldsymbol{x}_{Y_{l}, k}=\sum_{i=1}^{d} \boldsymbol{v}_{Y_{l}, k, i} s_{Y_{l}, k, i}=\boldsymbol{V}_{Y_{l}, k} \boldsymbol{s}_{Y_{l}, k}
$$


where $s_{Y_{l}, k, i}$ denotes the $i$-th transmitted data symbol from the $l$-th BS to its $k$-th user, $\boldsymbol{v}_{Y_{l}, k, i} \in C^{N^{t x} \times 1}$ is the unit-norm linear transmit beamforming vector for $s_{Y_{l}, k, i}, d\left(\leq \min \left(N^{t x}, N^{r x}\right)\right)$ is the total number of data symbols for each user, and $\boldsymbol{V}_{Y_{l}, k}=\left[\boldsymbol{v}_{Y_{l}, k, i}\right]_{1<i<d} \in \mathbb{C}^{N^{t x} \times d}$ is the transmit beamforming matrix at the $l$-th $\mathrm{BS}$ for transmitting the information-baring signal vector $\boldsymbol{s}_{Y_{l}, k}=\left[s_{Y_{l}, k, i}\right]_{1 \leq i \leq d}^{T} \in \mathbb{C}^{d \times 1}$ to its $k$-th UE, respectively. Hence, we can write

$$
\begin{aligned}
\boldsymbol{y}= & \underbrace{\left\|Y_{l^{*}}\right\|^{-\frac{\beta}{2}} \boldsymbol{G}_{Y_{l^{*}}} \boldsymbol{x}_{Y_{l^{*}}, k^{*}}}_{\text {intended signal }}+\underbrace{\left\|Y_{l^{*}}\right\|^{-\frac{\beta}{2}} \boldsymbol{G}_{Y_{l^{*}}} \sum_{k=1, k \neq k^{*}}^{U} \boldsymbol{x}_{Y^{*}, k}}_{\text {inter-user interference }} \\
& +\underbrace{\sum_{Y_{l} \in \Phi^{(b)} /\left\{Y_{\left.l^{*}\right\}}\right.}\left\|Y_{l}\right\|^{-\frac{\beta}{2}} \boldsymbol{G}_{Y_{l}} \sum_{k=1}^{U} \boldsymbol{x}_{Y_{l}, k}+\boldsymbol{\eta}}_{\text {inter-cell interference }}
\end{aligned}
$$

where $\boldsymbol{x}_{Y^{*}, k^{*}}$ denotes the intended transmit signal vector from the tagged BS to the reference UE.

With multiple receive antennas, each user in the system can simultaneously be given multiple data streams. Block diagonalization (BD) is a well-known linear transmit precoding technique for supporting multi-stream multiplexing. For the sake of notation, we denote the channel matrix from the $l$-th BS to its $k$-th UE with $\boldsymbol{G}_{Y_{l}, k} \in C^{N^{r x} \times N^{t x}}$. Let $\boldsymbol{G}_{Y_{l},(.)}^{\dagger}=$ $\left[\boldsymbol{G}_{Y_{l}, k}^{\dagger}\right]_{1 \leq k \leq U} \in C^{U N^{t x} \times N^{r x}}$ represent the concatenation of the channel matrices from the $l$-th BS to its $U$ UEs. Considering $\mathrm{BD}$, the transmit beamforming matrix from the $l$-th $\mathrm{BS}$ to its $k$-th UE is selected such that for all $j(\neq k) \in \mathcal{U}$, $\boldsymbol{G}_{Y_{l}, k} \boldsymbol{V}_{Y_{l, j}}=\mathbf{0}$, where $Y_{l} \in \Phi^{(b)}$ and $k \in \mathcal{U}$. The system can be converted into parallel (multi-user interference free) MIMO channels assuming $\boldsymbol{G}_{Y_{l}, \text { (.) }}$ is perfectly known, $d=N^{r x}$, and $N^{t x} \geq U N^{r x}$ [31]. There are however several drawbacks in utilizing $\mathrm{BD}$, examples include (i) additional complexity at the decoder considering the streams intended for each user are not generally aligned, and (ii) vulnerability to poor channel conditions due to the use of all channel dimensions for transmission [32].

Motivated by the above, in this paper, we employ a linear ZF transmit precoding where every BS (irrespective of $N^{r x}$ ) transmits a single spatial data stream to each of its respective UEs [33], [34]. This restriction is particularly justified for dense MIMO cellular systems with many multiple-antenna users such that $N^{t x} \ll U N^{r x}$. In fact, the results from the work in [32] indicate that the single-stream strategy with receive combining is preferable to $\mathrm{BD}$ in practice particularly due to its less stringent requirements in terms of receiver complexity and channel dependence. It is important to note that linear $\mathrm{ZF}$ and $\mathrm{BD}$ precoders are however identical when $N^{r x}=1$. Considering $d=1$, we can write $V_{Y_{l}, k}=\sqrt{P} \boldsymbol{v}_{Y_{l}, k}$ where $\boldsymbol{v}_{Y_{l}, k} \in C^{N^{t x} \times 1}$ is used to denote the unit-norm linear beamforming vector for the transmission of the information symbol from the $l$-th BS to its $k$-th, where $Y_{l} \in \Phi^{(b)}$ and $k \in \mathcal{U}$. We define $\boldsymbol{q}_{Y_{l}, k}=\boldsymbol{G}_{Y_{l}, k}^{\dagger} \boldsymbol{w}_{Y_{l}, k} \in C^{N^{t x} \times 1}$ to be the effective MISO channel from the $l$-th BS to its $k$-th user where $\boldsymbol{w}_{Y_{l}, k} \in \mathcal{C}^{N^{r x} \times 1}$ is a unit-norm linear combiner at the respective receiver. Note that under the assumption of i.i.d.
Rayleigh fading channel, $\boldsymbol{G}_{Y_{l}, k}$ is full rank with a probability one. In order to create zero inter-user interference, $\boldsymbol{v}_{Y_{l}, k}$, for $Y_{l} \in \Phi^{(b)}$ and $k \in \mathcal{U}$, is selected such that $\boldsymbol{q}_{Y_{l}, j}^{\dagger} \boldsymbol{v}_{Y_{l}, k}=0$ for all $j(\neq k) \in \mathcal{U}[34]$.

With multiple receive antennas at the user side, the combining filters can be selected in a way to cancel interference from nearby transmitters or/and to boost the received signal power. More specifically, considering the case where $N^{r x}=L U+\phi$, the linear ZF combiner may cancel the inter-cell interference from the nearest $L$ interferers conditioned on $N^{r x} \geq L U$, whilst there remains $\phi-1(\geq 0)$ degrees of freedom for improving the received signal strength. For example, this can be achieved at the reference UE by selecting $\boldsymbol{w}_{Y_{l^{*}, k^{*}}}$ on the nullspace of the effective interfering channel matrices $\boldsymbol{G}_{Y_{l}}, Y_{l} \in\left\{Y_{1}, \ldots, Y_{L}\right\}$, where $Y_{i}$ and $\left\|Y_{i}\right\|, i \in\{1,2, \ldots\}$, denote the location and Euclidean distance of the $i$-th closest interfering BS to the reference UE, respectively. We define $O$ where the columns form an orthonormal basis for this nullspace. Hence, the linear ZF filter is selected such that $\boldsymbol{w}_{Y_{l^{*}}, k^{*}}=O \frac{O^{\dagger} \boldsymbol{G}_{Y^{*}} \boldsymbol{v}_{Y^{*}, k^{*}}}{\left\|O^{\dagger} \boldsymbol{G}_{Y_{l^{*}}} \boldsymbol{v}_{Y_{l^{*}}, k^{*}}\right\|}$ [19]. By applying the linear ZF beamformer at the reference receiver, we can write

$$
\begin{aligned}
\boldsymbol{w}_{Y_{l^{*}}, k^{*}}^{\dagger} \boldsymbol{y}= & \sqrt{P}\left\|Y_{l^{*}}\right\|^{-\frac{\beta}{2}} \boldsymbol{w}_{Y_{l^{*}}, k^{*}}^{\dagger} \boldsymbol{G}_{Y_{l^{*}}} \boldsymbol{v}_{Y_{l^{*}}, k^{*} S Y_{l^{*}}, k^{*}} \\
& +\sum_{Y_{l} \in \hat{\Phi}^{(b)} /\left\{Y_{\left.l^{*}\right\}}\right.} \sqrt{P}\left\|Y_{l}\right\|^{-\frac{\beta}{2}} \boldsymbol{w}_{Y_{l^{*}, k^{*}}^{\dagger}}^{\dagger} \boldsymbol{G}_{Y_{l}} \sum_{k=1}^{U} \boldsymbol{v}_{Y_{l}, k} S_{Y_{l}, k} \\
& +\boldsymbol{w}_{Y_{l^{*}, k^{*}}^{\dagger} \boldsymbol{\eta}}^{\dagger}
\end{aligned}
$$

where $\hat{\Phi}^{(b)}=\Phi^{(b)} /\left\{Y_{1}, Y_{2}, \ldots, Y_{L}\right\}$. Note that linear $\mathrm{ZF}$ beamforming involves finding the pseudo-inverse of the multi-user MIMO channel which by the means of singular value decomposition (SVD) requires $O\left(24 N M^{2}+48 N^{2} M+54 N^{3}\right)$ floating point operations for an $N \times M(N \leq M)$ complex valued matrix [35].

The corresponding received intended signal power at the reference user can be expressed as

$$
X_{0}=P\left\|Y_{l^{*}}\right\|^{-\beta} h_{Y_{l^{*}}}
$$

where $h_{Y_{l^{*}}} \sim \operatorname{Gamma}(D, 1), D \triangleq\left(N^{t x}-U+1\right)\left(N^{r x}-L U\right)$. In addition, the post-processing aggregate network interference that the reference user experiences can be expressed as

$$
I_{\text {agg }}=P \sum_{Y_{l} \in \hat{\Phi}^{(b)} /\left\{Y_{\left.l^{*}\right\}}\right.}\left\|Y_{l}\right\|^{-\beta} h_{Y_{l}}
$$

where $h_{Y_{l}} \sim \operatorname{Gamma}(U, 1)$. Note that similar findings concerning the apprxomiation of the intended and interfering links using the Gamma distribution are presented in [23] and [28]. The corresponding signal-to-interferenceplus-noise ratio (SINR) of the reference user can therefore be formulated as

$$
\gamma=\frac{X_{0}}{I_{\mathrm{agg}}+\sigma^{2}}=\frac{\left\|Y_{l^{*}}\right\|^{-\beta} h_{Y_{l^{*}}}}{\sum_{Y_{l} \in \hat{\Phi}^{(b)} /\left\{Y_{\left.l^{*}\right\}}\right.}\left\|Y_{l}\right\|^{-\beta} h_{Y_{l}}+\frac{\sigma^{2}}{P}}
$$

where $\frac{\sigma^{2}}{P}$ is the reciprocal of the SNR. 
The downlink spectral efficiency in $\mathrm{b} / \mathrm{s} / \mathrm{Hz}$ of the cellular network with respect to the reference UE under consideration can be written as

$$
\mathcal{S}=\mathbb{E}\left\{\log _{2}(1+\gamma)\right\}
$$

By extending the main result from our previous work in [36] to a stochastic geometry-based multi-user MIMO setting, (11) can be expressed as in (12), shown at the bottom of this page. The conditional MGF of the intended signal power can be computed by [37]

$$
\mathcal{M}_{X_{0} \mid r_{0}}(z)=\mathcal{M}_{h_{l^{*}} \mid r_{0}}\left(z \operatorname{Pr}_{0}^{-\beta}\right)=\left(1+z \operatorname{Pr}_{0}^{-\beta}\right)^{-D} \text {. }
$$

In addition, a closed-form expression for the conditional MGF of the aggregate interference can be derived as in (14), shown at the bottom of this page, where $(a)$ follows by considering the interference in a disk of radius $\varrho\left(>r_{L}\right)$ with the limit as $\varrho \rightarrow+\infty ;(b)$ is from the associativity of multiplication for independent random variables; $(c)$ is written conditioned on $\mathcal{N}$ being the total number of interfering sources where $Y_{a}$ and $h_{Y_{a}}$ are used to respectively denote the location and channel from an arbitrary BS $a$ to the reference user; $(d)$ follows from characterizing $\mathcal{N}$ with a Binomial distribution with parameters $(\rho, \kappa)$; with the pdf of the distance of uniformly-distributed arbitrary node to the reference user

$$
\mathcal{P}_{\left\|Y_{a}\right\|}(r)=\frac{2 r}{\varrho^{2}-r_{L}^{2}}, \quad r_{L}<r<\varrho
$$

and the following integral identity $(\alpha \geq 0, \beta>2)$

$$
\begin{aligned}
\mathbb{E}_{\left\|Y_{a}\right\|} & \left\{\exp \left(-\alpha\left\|Y_{a}\right\|^{-\beta}\right)\right\} \\
= & \frac{2 \alpha^{\frac{2}{\beta}}}{\beta\left(\varrho^{2}-r_{L}^{2}\right)} \\
& \times\left[\Gamma\left(\frac{-2}{\beta}, \alpha \varrho^{-\beta}\right)-\Gamma\left(\frac{-2}{\beta}, \alpha r_{L}^{-\beta}\right)\right]
\end{aligned}
$$

(e) can be derived by simultaneously taking the limits as $\varrho \rightarrow+\infty, \kappa \rightarrow+\infty, \rho \rightarrow 0$, and utilizing the Poisson limit theorem with $\frac{\kappa \rho}{\pi \varrho^{2}}=\lambda^{(b)}<\infty$; finally, $(f)$ is obtained by taking the average with respect to $h_{Y_{a}}$ using the integral identities $(\alpha \geq 0, \beta>2)$

$$
\begin{aligned}
\mathbb{E}_{h_{Y_{a}}}\left\{h_{Y_{a}}^{\frac{2}{\beta}}\right\} & \int_{0}^{+\infty} \frac{h^{U+\frac{2}{\beta}-1}}{\Gamma(U)} \exp (-h) \mathrm{d} h=\frac{\Gamma\left(U+\frac{2}{\beta}\right)}{\Gamma(U)}, \\
= & \mathbb{E}_{h_{Y_{a}}}\left\{\Gamma\left(1-\frac{2}{\beta}, \alpha h_{Y_{a}}\right) h_{Y_{a}}^{\frac{2}{\beta}}\right\} \\
= & \int_{0}^{+\infty} \Gamma\left(1-\frac{2}{\beta}, \alpha h\right) \times \frac{h^{U+\frac{2}{\beta}-1}}{\Gamma(U)} \exp (-h) \mathrm{d} h \\
= & \frac{{ }_{2} F_{1}\left(U+1, U+\frac{2}{\beta} ; U+\frac{2}{\beta}+1 ;-\frac{1}{\alpha}\right)}{\left(1+\frac{2}{U \beta}\right) \alpha^{\frac{2}{\beta}+U}},
\end{aligned}
$$

$$
\begin{aligned}
& S=\log _{2}(e) \int_{0}^{+\infty} \int_{r_{0}}^{+\infty} \int_{0}^{+\infty} \frac{\mathcal{M}_{I_{\mathrm{agg}} \mid r_{L}}(z)\left(1-\mathcal{M}_{X_{0} \mid r_{0}}(z)\right)}{z} \exp \left(-z \sigma^{2}\right) \mathcal{P}_{\left\|Y_{L}\right\| \mid \| Y_{l^{*} \|}}\left(r_{L} \mid r_{0}\right) \mathcal{P}_{\left\|Y_{l^{*} \|}\right\|}\left(r_{0}\right) \mathrm{d} z \mathrm{~d} r_{L} \mathrm{~d} r_{0} \\
& \mathcal{M}_{I_{\mathrm{agg}} \mid r_{L}}(z)=\mathbb{E}\left\{\exp \left(-z P \sum_{Y_{l} \in \hat{\Phi}^{(b)} /\left\{Y_{\left.l^{*}\right\}}\right.}\left\|Y_{l}\right\|^{-\beta} h_{Y_{l}}\right)\right\} \\
& \stackrel{(a)}{=} \lim _{\varrho \rightarrow \infty} \mathbb{E}\left\{\exp \left(-z P \sum_{Y_{l} \in \hat{\Phi}^{(b)} /\left\{Y_{\left.l^{*}\right\}}\right.}\left\|Y_{l}\right\|^{-\beta} h_{Y_{l}}\right)\right\} \\
& \stackrel{(b)}{=} \lim _{\varrho \rightarrow \infty} \mathbb{E}\left\{\prod_{Y_{l} \in \hat{\Phi}^{(b)} /\left\{Y_{\left.l^{*}\right\}}\right.} \exp \left(-z P\left\|Y_{l}\right\|^{-\beta} h_{Y_{l}}\right)\right\} \\
& \stackrel{(c)}{=} \lim _{\varrho \rightarrow \infty} \mathbb{E}_{\mathcal{N}}\left\{\left(\mathbb{E}_{\left\|Y_{a}\right\|, h_{Y_{a}}}\left\{\exp \left(-z P\left\|Y_{a}\right\|^{-\beta} h_{Y_{a}}\right)\right\}\right)^{\mathcal{N}}\right\} \\
& \stackrel{(d)}{=} \lim _{\varrho \rightarrow \infty}\left(\rho\left(\mathbb{E}_{\left\|Y_{a}\right\|, h_{Y_{a}}}\left\{\exp \left(-z P\left\|Y_{a}\right\|^{-\beta} h_{Y_{a}}\right)\right\}-1\right)+1\right)^{\kappa} \\
& \stackrel{(e)}{=} \exp \left(-\pi \lambda^{(b)} \mathbb{E}_{h_{Y_{a}}}\left\{\left[\Gamma\left(1-\frac{2}{\beta}\right)-\Gamma\left(1-\frac{2}{\beta}, z \operatorname{Pr}_{L}^{-\beta} h_{Y_{a}}\right)\right]\left(z \operatorname{Ph}_{Y_{a}}\right)^{\frac{2}{\beta}}+r_{L}^{2}\left(\exp \left(-z \operatorname{Pr}_{L}^{-\beta} h_{Y_{a}}\right)-1\right)\right\}\right) \\
& \stackrel{(f)}{=} \exp \left(-\pi \lambda^{(b)}\left[r_{L}^{2}\left(\left(z \operatorname{Pr}_{L}^{-\beta}+1\right)^{-U}-1\right)+\frac{\Gamma\left(U+\frac{2}{\beta}\right)}{(z P)^{U} \Gamma(U)}\left((z P)^{U+\frac{2}{\beta}} \Gamma\left(1-\frac{2}{\beta}\right)-U \Gamma(U) r_{L}^{U \beta+2}\right.\right.\right. \\
& \left.\left.\left.\times{ }_{2} \tilde{F}_{1}\left(U+1, U+\frac{2}{\beta} ; U+\frac{2}{\beta}+1 ;-\frac{r_{L}^{\beta}}{z P}\right)\right)\right]\right)
\end{aligned}
$$


and

$$
\begin{aligned}
\mathbb{E}_{h_{Y_{a}}}\left\{\exp \left(-\alpha h_{Y_{a}}\right)\right\} & =\int_{0}^{+\infty} \frac{h^{U-1}}{\Gamma(U)} \exp (-h(1+\alpha)) \mathrm{d} h \\
& =\frac{1}{(1+\alpha)^{U}} .
\end{aligned}
$$

\section{Heterogeneous Cellular Networks}

In this section, we extend the analysis from the previous section on MIMO single-tier cellular networks to the generalized case with multi-antenna heterogeneous sources. Due to the similarities, only key technical details are highlighted in what follows.

Here, we consider the downlink of a multi-tier MIMO cellular network where $T$ classes of BSs are deployed according to stationary PPPs $\Phi_{t}^{(b)}$ with spatial densities $\lambda_{t}^{(b)}$, where $t \in \mathcal{T}=\{1,2, \ldots, T\}$. The tiers of BSs are assumed to further differ in terms of number of transmit antennas $N_{t}^{t x}$, per-user transmit power $P_{t}$, artificial-bias $\varpi_{t}$, and number of users served in each resource block $U_{t}$ [23].

A fundamental difference between multi-antenna homogeneous and heterogeneous cellular networks is in the cellular association strategy. Specifically, in the latter, in contrast to SISO HetNets, connecting the user to the BS that provides the greatest received signal power does not strictly result in the best SINR. The work in [38] investigates flexible cell selection in MISO HetNets and illustrates that by appropriate artificial-biasing of the coverage range of different tiers, a tight approximation on the strategy for maximizing SINR can be derived. Here, we extend the cellular association strategy proposed in [38] to the generalized case with multiple receive antennas at the UEs.

Specifically, the cell selection strategy under consideration is concerned with connecting the user to the closest BS $l^{*}$ of a certain tier $t^{*}$ located at $Y_{t^{*}, l^{*}}$ which provides the greatest biased received signal power. This can be mathematically formulated as

$$
Y_{t^{*}, l^{*}}=\arg \max \left(\varpi_{t} P_{t} D_{t}\left\|Y_{t, l}\right\|^{-\beta}\right), \quad t \in \mathcal{T}, Y_{t, l} \in \Phi_{t}^{(b)}
$$

where $D_{t} \triangleq\left(N_{t}^{t x}-U_{t}+1\right)\left(N^{r x}-L_{t} U_{t}\right)$ represents the postprocessing gain provided via linear $\mathrm{ZF}$ transmit precoding and receive combining from the tier- $t$ BSs to the reference UE. Through adopting a similar approach to that in [39], the cellular association probability to the closest BS $l^{*}$ from the tier- $t^{*}$ network is given by (21), shown at the bottom of this page. The probability inside the integral in (21) denotes the likelihood that no tier- $t$ BS is closer to the reference UE than $\left(\frac{w_{t} * P_{t^{*}} D_{t^{*}}}{w_{t} P_{t} D_{t}}\right) r_{t^{*}, 0}$ and is computed by (22), shown at the bottom of this page, where $C(0, r)$ is defined as a circle of radius $r$ centered at the origin on the Euclidean plane. Therefore, the respective tier connection probability can be written as

$$
\varphi_{t^{*}}=\frac{\lambda_{t^{*}}^{(b)}}{\sum_{t \in \mathcal{T}} \lambda_{t}^{(b)}\left(\frac{\varpi_{t} P_{t} D_{t}}{\varpi_{t^{*}} P_{t^{*}} D_{t^{*}}}\right)^{\frac{2}{\beta}} .}
$$

The respective spectral efficiency of a typical user in the MIMO multi-tier cellular network can be expressed as [20]

$$
\mathcal{S}=\sum_{t^{*} \in \mathcal{T}} \mathbb{E}\left\{\log _{2}\left(1+\gamma_{t^{*}}\right)\right\} \varphi_{t^{*}}
$$

where $\gamma_{t^{*}}$ is the instantaneous SINR at the reference UE from the tagged $l^{*}$-th tier- $t^{*}$ BS. In practice, the user in the HetNet paradigm can estimate the channels from the BSs of different tiers in order to decide on an interference cancellation policy which results in the best performance. For the sake of analytical tractability, here, we consider a sub-optimal policy where the user can cancel interfering signals from the intra-tier sources only. Specifically, considering perfect CSI from the intra-tier sources is available, with linear ZF combining at the respective receiver, the interference from the nearest $L_{t^{*}}$ tier- $t^{*}$ interferers can be mitigated conditioned on $N^{r x}>L_{t^{*}} U_{t^{*}}$. The post-processing aggregate network interference can therefore be written as

$$
\begin{aligned}
I_{t^{*}, \text { agg }}= & \underbrace{\sum_{Y_{t^{*}, l} \in \hat{\Phi}_{t^{*}}^{(b)} /\left\{Y_{\left.t^{*}, l^{*}\right\}}\right.} P_{t^{*}}\left\|Y_{t^{*}, l}\right\|^{-\beta} h_{Y_{t^{*}, l}}}_{\text {post-processed intra-tier interference }} \\
& +\underbrace{\sum_{t \in \mathcal{T} /\left\{t^{*}\right\}, Y_{t, l} \in \Phi_{t}^{(b)}} P_{t}\left\|Y_{t, l}\right\|^{-\beta} h_{Y_{t, l}}}_{\text {inter-tier interference }}
\end{aligned}
$$

$$
\begin{aligned}
& \varphi_{t^{*}}=\mathscr{P}\left(\bigcap_{t^{*} \in \mathcal{T}, Y_{t^{*}, l^{*}} \in \Phi_{t^{*}}^{(b)} \neq t \in \mathcal{T}, Y_{t, l} \in \Phi_{t}^{(b)}}\left\{\left\|Y_{t, l}\right\|>\left(\frac{\varpi_{t^{*}} P_{t^{*}} D_{t^{*}}}{\varpi_{t} P_{t} D_{t}}\right)\left\|Y_{t^{*}, l^{*}}\right\|\right\}\right) \\
& =\prod_{t^{*} \in \mathcal{T}, Y_{t^{*}, l^{*}} \in \Phi_{t^{*}}^{(b)} \neq t \in \mathcal{T}, Y_{t, l} \in \Phi_{t}^{(b)}} \int_{0}^{+\infty} \mathscr{P}\left(\left\|Y_{t, l}\right\|>\left(\frac{\varpi_{t^{*}} P_{t^{*}} D_{t^{*}}}{\varpi_{t} P_{t} D_{t}}\right) r_{t^{*}, 0}\right) \mathcal{P}_{\| Y_{t^{*}, l^{*} \|}}\left(r_{t^{*}, 0}\right) \mathrm{d} r_{t^{*}, 0} \\
& \mathscr{P}\left(\left\|Y_{t, l}\right\|>\left(\frac{\varpi_{t^{*}} P_{t^{*}} D_{t^{*}}}{\varpi_{t} P_{t} D_{t}}\right) r_{t^{*}, 0}\right)=\mathscr{P}\left(\Phi_{t}^{(b)} \bigcap C\left(0,\left(\frac{\varpi_{t^{*}} P_{t^{*}} D_{t^{*}}}{\varpi_{t} P_{t} D_{t}}\right) r_{t^{*}, 0}\right)=\{\}\right) \\
& =\exp \left(-\pi \lambda_{t}^{(b)}\left(\frac{\varpi_{t^{*}} P_{t^{*}} D_{t^{*}}}{\varpi_{t} P_{t} D_{t}}\right)^{2} r_{t^{*}, 0}^{2}\right)
\end{aligned}
$$




$$
\begin{aligned}
& \gamma_{t^{*}}=\frac{X_{t^{*}, 0}}{I_{t^{*}, \mathrm{agg}}+\sigma^{2}}=\frac{P_{t^{*}}\left\|Y_{t^{*}, l^{*}}\right\|^{-\beta} h_{Y_{t^{*}, l^{*}}}}{\sum_{Y_{t^{*}, l} \in \hat{\Phi}_{t^{*}}^{(b)} /\left\{Y_{\left.t^{*}, l^{*}\right\}}\right.} P_{t^{*}}\left\|Y_{t^{*}, l}\right\|^{-\beta} h_{t_{t^{*}, l}}+\sum_{t \in \mathcal{T} /\left\{t^{*}\right\}, Y_{t, l} \in \Phi_{t}^{(b)}} P_{t}\left\|Y_{t, l}\right\|^{-\beta} h_{Y_{t, l}}+\sigma^{2}}
\end{aligned}
$$

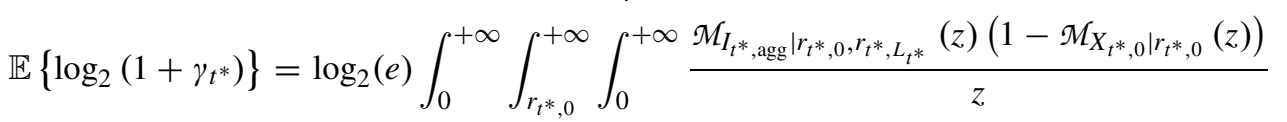

$$
\begin{aligned}
& \times \exp \left(-z \sigma^{2}\right) \mathcal{P}_{\left\|Y_{t^{*}, L_{t^{*}}}\right\| \mid \| Y_{t^{*}, 0 \|}}\left(r_{t^{*}, L_{t^{*}}} \mid r_{t^{*}, 0}\right) \mathcal{P}_{\left\|Y_{t^{*}, 0}\right\|}\left(r_{t^{*}, 0}\right) \mathrm{d} z \mathrm{~d} r_{t^{*}, L_{t^{*}}} \mathrm{~d} r_{t^{*}, 0}
\end{aligned}
$$

where $h_{Y_{t, l}}$ denotes the channel fading power gain from the $l$-th tier- $t$ BS located at $Y_{t, l}$ and $\hat{\Phi}_{t^{*}}^{(b)}=$ $\Phi_{t^{*}}^{(b)} /\left\{Y_{t^{*}, 1}, Y_{t^{*}, 2}, \ldots, Y_{t^{*}, L_{t}}\right\}$ with $Y_{t, i}, t \in \mathcal{T}, i \in\{1,2, \ldots\}$, being used to denote the location of the $i$-th closest interfering tier- $t$ BS to the reference UE. Hence, we have (26), shown at the top of this page, where $h_{Y_{t^{*}, l^{*}}} \sim \operatorname{Gamma}\left(D_{t^{*}}, 1\right)$ and $h_{Y_{t, l}} \sim \operatorname{Gamma}\left(U_{t}, 1\right), t \in \mathcal{T}$. The respective user spectral efficiency in $\mathrm{b} / \mathrm{s} / \mathrm{Hz}$ can therefore be expressed as in (27), shown at the top of this page, where

$$
\begin{aligned}
\mathcal{P}_{\| Y_{t^{*}, l^{*} \|}}\left(r_{t^{*}, 0}\right)= & \frac{2 \pi \lambda_{t^{*}}^{(b)} r_{t^{*}, 0}}{\varphi_{t^{*}}} \\
& \times \exp \left(-\pi r_{t^{*}, 0}^{2} \sum_{t \in \mathcal{T}} \lambda_{t}^{(b)}\left(\frac{\varpi_{t} P_{t} D_{t}}{\varpi_{t^{*}} P_{t^{*}} D_{t^{*}}}\right)^{\frac{2}{\beta}}\right)
\end{aligned}
$$

and

$$
\begin{aligned}
\mathcal{P}_{\left\|Y_{t^{*}, L_{t^{*}}}\right\| \mid\left\|Y_{t^{*}, 0 \|}\right\|}\left(r_{t^{*}, L_{t^{*}}} \mid r_{t^{*}, 0}\right) \\
=\frac{2 \pi \lambda_{t^{*}}^{(b)} r_{t^{*}, L_{t^{*}}}}{\Gamma\left(L_{t^{*}}\right)}\left(\pi\left(r_{t^{*}, L_{t^{*}}}^{2}-r_{t^{*}, 0}^{2}\right) \lambda_{t^{*}}^{(b)}\right)^{L_{t^{*}-1}} \\
\quad \times \exp \left(-\pi\left(r_{t^{*}, L_{t^{*}}}^{2}-r_{t^{*}, 0}^{2}\right) \lambda_{t^{*}}^{(b)}\right) .
\end{aligned}
$$

The conditional statistics of the intended signal power can be calculated using [37]

$$
\begin{aligned}
\mathcal{M}_{X_{t^{*}, 0} \mid r_{t^{*}, 0}}(z) & =\mathcal{M}_{h_{t^{*}, l^{*}} \mid r_{t^{*}, 0}}\left(z P_{t^{*} r_{t^{*}, 0}}^{-\beta}\right) \\
& =\left(1+z P_{t^{*}} r_{t^{*}, 0}^{-\beta}\right)^{-D_{t^{*}}} .
\end{aligned}
$$

Furthermore, by leveraging on the independence property of PPP and the assumption on uncorrelated channel statistics, the MGF of the aggregate network interference can be written as in (31), shown at the bottom of this page. Accordingly, the post-processing intra-tier interference can be derived directly based on the result from the previous section as in (32), shown at the bottom of this page.

We derive a closed-form expression for the conditional statistics of the inter-tier interference in (33), shown at the top of the next page, where (a) shows the interference from independent PPPs in a disk of radius $\varrho(\rightarrow+\infty)$; (b) is formed by conditioning on $\mathcal{N}_{t}$ as the number of interfering tier- $t$ sources where $Y_{t, a}$ and $h_{Y_{t, a}}$ respectively represent the location and channel from an arbitrary $a$-th tier- $t$ BS with respect the reference UE; $(c)$ is from characterizing $\mathcal{N}_{t}$ as a Binomial random variable with parameters $\left(\rho_{t}, \kappa_{t}\right)$; with the pdf of the distance of uniformlydistributed interferer in the disk of radius $\varrho$ with respect to the

$$
\begin{aligned}
& \mathcal{M}_{I^{*}, \text { agg }} \mid r_{t^{*}, 0}, r_{t^{*}, L}(z)=\mathbb{E}\left\{\exp \left(-z\left(\sum_{Y_{t^{*}, l} \in \hat{\Phi}_{t^{*}}^{(b)} /\left\{Y_{t^{*}, l^{*}}\right\}} P_{t^{*}}\left\|Y_{t^{*}, l}\right\|^{-\beta} h_{Y_{t^{*}, l}}+\sum_{t \in \mathcal{T} /\left\{t^{*}\right\}, Y_{t, l} \in \Phi_{t}^{(b)}} P_{t}\left\|Y_{t, l}\right\|^{-\beta} h_{Y_{t, l}}\right)\right)\right\} \\
& =\mathbb{E}\left\{\exp \left(-z \sum_{Y_{t^{*}, l} \in \hat{\Phi}_{t^{*}}^{(b)} /\left\{Y_{\left.t^{*}, l^{*}\right\}}\right.} P_{\left.t^{*}\left\|Y_{t^{*}, l}\right\|^{-\beta} h_{Y_{t^{*}, l}}\right)}\right)\right\} \mathbb{E}\left\{\exp \left(-z \sum_{t \in \mathcal{T} /\left\{t^{*}\right\}, Y_{t, l} \in \Phi_{t}^{(b)}} P_{t}\left\|Y_{t, l}\right\|^{-\beta} h_{Y_{t, l}}\right)\right\} \\
& =\mathcal{M}_{I_{t^{*}, \text { intra }}} \mid r_{t^{*}, L_{t^{*}}}(z) \mathcal{M}_{I_{t^{*} \text {,inter }} \mid r_{t^{*}, 0}}(z) \\
& \mathcal{M}_{I^{*}, \text { intra }} r_{t^{*}, L_{t^{*}}}(z)=\exp \left(-\pi \lambda_{t^{*}}^{(b)}\left[r_{L_{t^{*}}}^{2}\left(\left(z P_{t^{*}} r_{L_{t^{*}}}^{-\beta}+1\right)^{-U_{t^{*}}}-1\right)+\frac{\Gamma\left(U_{t^{*}}+\frac{2}{\beta}\right)}{\left(z P_{t^{*}}\right)^{U_{t^{*}}} \Gamma\left(U_{t^{*}}\right)}\left(\left(z P_{t^{*}}\right)^{U_{t^{*}+\frac{2}{\beta}}} \Gamma\left(1-\frac{2}{\beta}\right)\right.\right.\right. \\
& \left.\left.\left.-U_{t^{*} \Gamma}\left(U_{t^{*}}\right) r_{L_{t^{*}}}^{U_{t^{*} \beta+2}}{ }_{2} \tilde{F}_{1}\left(U_{t^{*}}+1, U_{t^{*}}+\frac{2}{\beta} ; U_{t^{*}}+\frac{2}{\beta}+1 ;-\frac{r_{L_{t^{*}}}^{\beta}}{z P_{t^{*}}}\right)\right)\right]\right)
\end{aligned}
$$




$$
\begin{aligned}
& \mathcal{M}_{I^{*}, \text { inter }} r_{t^{*}, 0}(z) \stackrel{(a)}{=} \lim _{\varrho \rightarrow \infty} \prod_{t \in \mathcal{T} /\left\{t^{*}\right\}} \mathbb{E}_{\left\|Y_{t, l}\right\|, h_{Y_{t, l}}}\left\{\prod_{Y_{t, l} \in \Phi_{t}^{(b)}} \exp \left(-z P_{t}\left\|Y_{t, l}\right\|^{-\beta} h_{Y_{t, l}}\right)\right\} \\
& \stackrel{(b)}{=} \lim _{\varrho \rightarrow \infty} \prod_{t \in \mathcal{T} /\left\{t^{*}\right\}} \mathbb{E}_{\mathscr{N}_{t}}\left\{\left(\mathbb{E}_{\left\|Y_{t, a}\right\|, h_{Y_{t, a}}}\left\{\exp \left(-z P_{t}\left\|Y_{t, a}\right\|^{-\beta} h_{Y_{t, a}}\right)\right\}\right)^{\mathcal{N}_{t}}\right\} \\
& \stackrel{(c)}{=} \lim _{\varrho \rightarrow \infty} \prod_{t \in \mathcal{T} /\left\{t^{*}\right\}}\left(\rho_{t}\left(\mathbb{E}_{\left\|Y_{t, a}\right\|, h_{Y_{t, a}}}\left\{\exp \left(-z P_{t}\left\|Y_{t, a}\right\|^{-\beta} h_{Y_{t, a}}\right)\right\}-1\right)+1\right)^{\kappa_{t}} \\
& \stackrel{(d)}{=} \prod_{t \in \mathcal{T} /\left\{t^{*}\right\}} \exp \left(-\pi \lambda_{t}^{(b)} \mathbb{E}_{h_{Y_{t, a}}}\left\{\left[\Gamma\left(1-\frac{2}{\beta}\right)-\Gamma\left(1-\frac{2}{\beta}, z P_{t} \Psi_{t}^{-\beta} h_{Y_{t, a}}\right)\right]\right.\right. \\
& \left.\left.\times\left(z P_{t} h_{Y_{t, a}}\right)^{\frac{2}{\beta}}+\Psi_{t}^{2}\left(\exp \left(-z P_{t} \Psi_{t}^{-\beta} h_{Y_{t, a}}\right)-1\right)\right\}\right) \\
& \stackrel{(e)}{=} \exp \left(-\pi \sum_{t \in \mathcal{T} /\left\{t^{*}\right\}} \lambda_{t}^{(b)}\left[\Psi_{t}^{2}\left(\left(z P_{t} \Psi_{t}^{-\beta}+1\right)^{-U_{t}}-1\right)+\frac{\Gamma\left(U_{t}+\frac{2}{\beta}\right)}{\left(z P_{t}\right)^{U_{t}} \Gamma\left(U_{t}\right)}\left(\left(z P_{t}\right)^{U_{t}+\frac{2}{\beta}} \Gamma\left(1-\frac{2}{\beta}\right)\right.\right.\right. \\
& \left.\left.\left.-U_{t} \Gamma\left(U_{t}\right) \Psi_{t}^{U_{t} \beta+2}{ }_{2} \tilde{F}_{1}\left(U_{t}+1, U_{t}+\frac{2}{\beta} ; U_{t}+\frac{2}{\beta}+1 ;-\frac{\Psi_{t}^{\beta}}{z P_{t}}\right)\right)\right]\right)
\end{aligned}
$$

origin

$$
\mathcal{P}_{\left\|Y_{t, a}\right\|}(r)=\frac{2 r}{\varrho^{2}-\Psi_{t}^{2}}, \quad \Psi_{t}<r<\varrho
$$

where

$$
\Psi_{t}=\left(\frac{\varpi_{t} P_{t} D_{t}}{\varpi_{t^{*}} P_{t^{*}} D_{t^{*}}}\right)^{\frac{1}{\beta}} r_{t^{*}, 0}
$$

and utilizing the integral identity in (16), $(d)$ is obtained by by simultaneously taking the limits as $\varrho \rightarrow+\infty, \kappa_{t} \rightarrow+\infty$, $\rho_{t} \rightarrow 0$, and utilizing the Poisson limit theorem with $\frac{\kappa_{t} \rho_{t}}{\pi \varrho^{2}}=\lambda_{t}^{(b)}<\infty$; finally, $(e)$ is obtained by taking the average with respect to $h_{Y_{t, a}}$ using the integral identities in (17), (18), and (19).

It is important to note that although the spectral efficiency of a typical user can unveil important design guidelines, it does not directly capture the overall downlink performance where different number of users may be served at a time. In order to take this into account, we can calculate the area spectral efficiency $\mathcal{A}$ in $\mathrm{b} / \mathrm{s} / \mathrm{Hz} / \mathrm{km}^{2}$. For the sake of analytical tractability, we limit our area spectral efficiency analysis to two different full-SDMA $\left(U_{t}=N_{t}^{t x}, t \in \mathcal{T}\right)$ and SUBF $\left(U_{t}=1, t \in \mathcal{T}\right)$ transmission schemes. For the generalized MIMO HetNets, this can be mathematically formulated as

$$
\mathcal{A}=\sum_{t^{*} \in \mathcal{T}} \lambda_{t^{*}}^{(b)} U_{t^{*}} \mathbb{E}\left\{\log _{2}\left(1+\gamma_{t^{*}}\right)\right\} \varphi_{t^{*}}
$$

where $\lambda_{t^{*}}^{(b)}$ is the deployment density of tier- $t^{*}$ BSs per $\mathrm{km}^{2}$.

It should be noted that the spectral efficiency functions derived are highly non-linear involving multiple improper integrals. Hence, exact closed-form expressions even for simplified cases cannot be obtained. However, the proposed approach together with the systematic methodology for deriving intended signal power and aggregate network interference conditional statistics in closed-form greatly simplifies system analysis and optimization as illustrated in the next section.

\section{Performance Evaluation}

In this section, we present several theoretical and simulation studies in order to examine the validity of the proposed analytical framework. Furthermore, we aim to quantify the impact of different system parameters and decisions on achievable performance which unveils important insights on cellular network design. In order to achieve this, Monte-Carlo trials with Gamma-distributed intended and interfering channel power gains are employed. It should be highlighted that the results based on stochastic geometry theory are shown to be worst-case representations to those obtained from systemlevel simulations with the entire transmit-receive processing chain [40].

\section{A. Monte-Carlo Simulations}

The underlying steps to evaluate the spectral efficiency of a typical UE in generalized multi-antenna multi-tier cellular networks with linear ZF transmit and receive beamforming using Monte-Carlo simulations are described below.

1) Initialize the set of parameters for each tier- $t$ network, including deployment density $\lambda_{t}^{(b)}$, number of transmit antennas $N_{t}^{t x}$, per-user transmit power $P_{t}$, number of users served in each resource block $U_{t}$, number of receive antennas to be used for interference cancellation $L_{t}$, and artificial-biasing weight $\varpi_{t}$.

2) Set the noise power $\sigma^{2}$, path-loss exponent $\beta$, and receive antennas $N^{r x}$ at the UEs.

3) Define a circle of radius $d$ and hence area $\pi d^{2}$ around a reference UE located at the origin.

4) For each tier- $t$ network, generate the statistical number of BSs $\mathcal{N}_{t}^{(b)} \sim \operatorname{Poisson}\left(\pi d^{2} \lambda_{t}^{(b)}\right)$, then deploy uniformlydistributed heterogeneous BSs within the circular region of area $\pi d^{2}$. 
Homogeneous Cellular Network

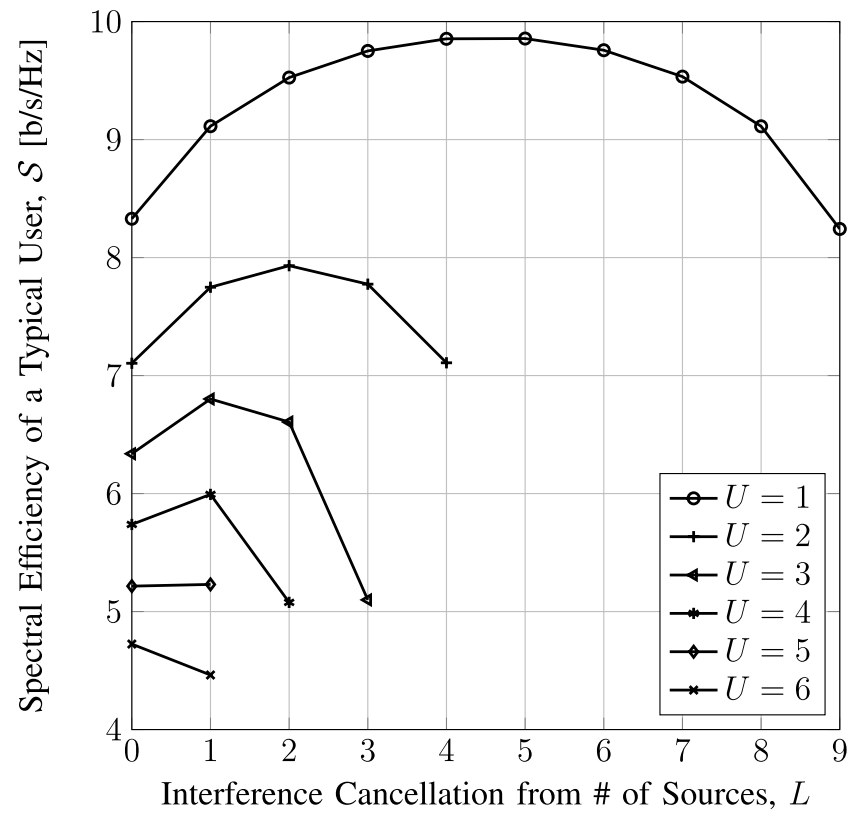

Fig. 1. System parameters are: $\lambda^{(b)}=0.05, N^{t x}=10$, $N^{r x}=10, \mathrm{SNR}=80 \mathrm{~dB}, \beta=4$.

5) Generate independent channel power gains from all deployed BSs to the reference UE.

6) Associate the reference UE to the closest BS of a particular tier which provides the strongest biased received signal power; thus finding the intended signal power $X$.

7) Compute the post-processing aggregate network interference on the reference UE $I_{\text {agg }}$ using the sum of received signal powers from all remaining interfering links.

8) Calculate the SINR of the reference UE by dividing $X$ with $I_{\text {agg }}+\sigma^{2}$ and thereafter evaluate the ergodic rate.

9) Spectral efficiency of the reference user is obtained by repeating steps 4-8 a sufficiently large number of times and then taking the average.

In the simulation results presented in this paper, the trials were conducted for $50 \mathrm{k}$ times over a radius of $20 \mathrm{~km}$. Note that the execution time for obtaining a typical simulation curve using a standard workstation at present time can range from a couple of days to more than a week depending on the system settings. With the proposed analytical framework, on the other hand, similar results can be produced in the order of minutes, thus highlighting the underlying advantages in terms of computational complexity. Henceforth, theoretical and simulation results are respectively depicted in the figures by solid/dashed lines and markers.

\section{B. Single-Tier Cellular Networks}

In Fig. 1, the spectral efficiency of a typical user in a $10 \times 10$ MIMO system is depicted with varying number of UEs served per resource block and different uses of receive antennas for boosting the signal power or/and cancelling the interference from nearby sources. In addition, the impact of path-loss severity on spectral efficiency of a typical user considering different configurations of receive antennas is

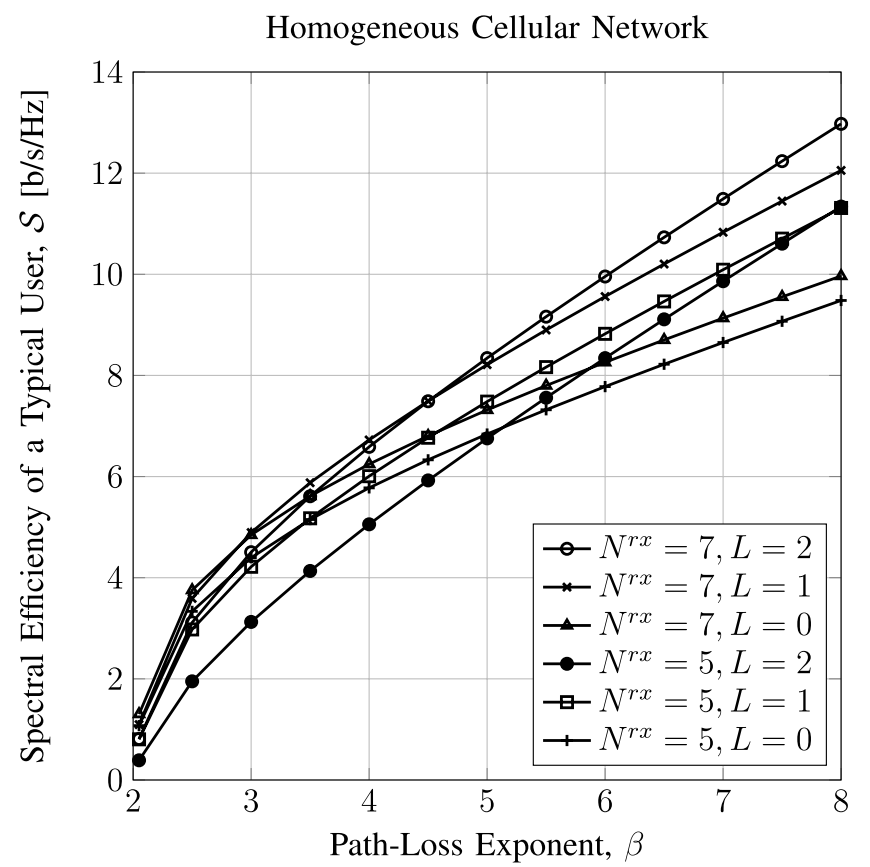

Fig. 2. System parameters are: $\lambda^{(b)}=0.1, N^{t x}=8, U=2$, $\mathrm{SNR}=100 \mathrm{~dB}$.

illustrated in Fig. 2. The reference user performance is shown to improve with a lower number of simultaneously served users due to the added degrees of freedom at the transmitter side as well as the reduced interference from the other BSs. For example, from Fig. 1, with $L=0$ (full-diversity), the reference user spectral efficiency with $U=1$ (SUBF) and $U=6$ (SDMA) are recorded to be around $8.33 \mathrm{~b} / \mathrm{s} / \mathrm{Hz}$ and $4.72 \mathrm{~b} / \mathrm{s} / \mathrm{Hz}$, respectively; the improvement however comes at the cost of diminished multiplexing gain. Trivially, from Fig. 2 , the rate performance is shown to improve somewhat linearly as the path-loss exponent for all intended and interfering links is increased. Overall, the validity of the proposed analytical framework is confirmed given there is a near-exact fit between the theoretical results and those from the Monte-Carlo trials.

Furthermore, the optimal trade-off between signal power enhancement and interference cancellation at the receiver is shown to depend on certain system parameters. For a given number of transmit and receive antennas, the interference cancellation from an optimal number of sources $\left(L^{*}\right)$ which maximizes the reference user spectral efficiency decreases as the number of UEs requesting service is increased. The performance penalty from using more (less) receive antennas than optimal towards diversity is greater (smaller) when a lower (higher) number of users are present - the converse of this trend on diversity holds for interference cancellation. In addition, the performance gain from signal power enhancement is greater (smaller) than that from interference cancellation under low (high) path-loss exponents. With more receive antennas at the UEs, as $\beta$ is increased, the rate at which using additional receive antennas for interference cancellation becoming favourable over further boosting of the intended signal power increases. For instance, from Fig. 2, with $N^{t x}=8$, $\beta=5$, and $U=2$, the optimal number of nearby sources 


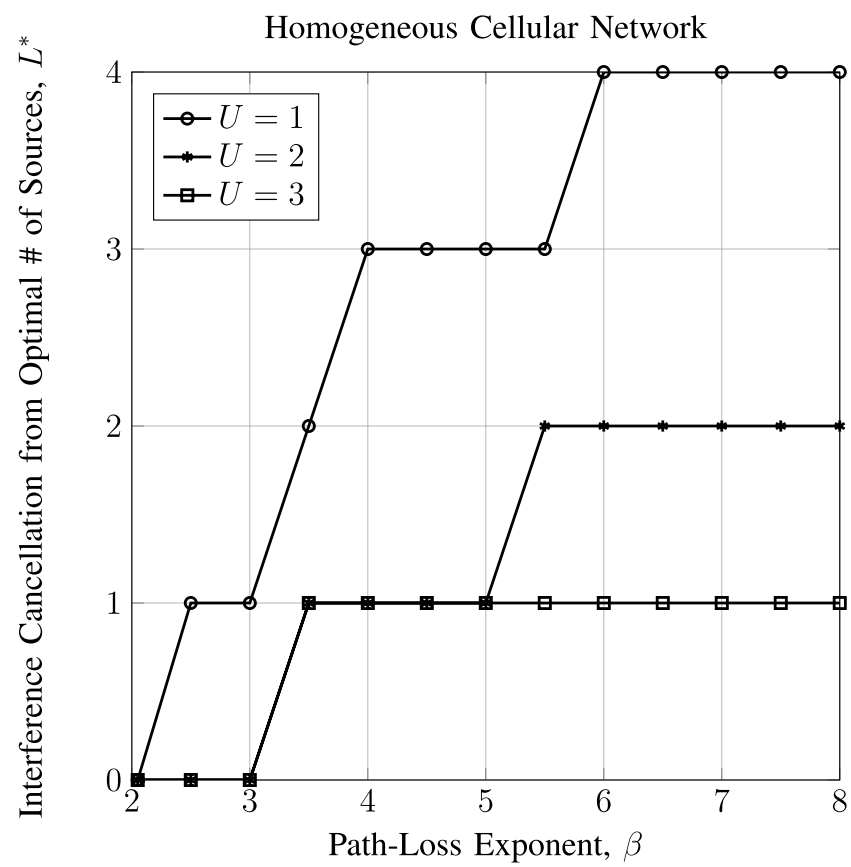

Fig. 3. System parameters are: $\lambda^{(b)}=0.2, N^{t x}=12, N^{r x}=6, \sigma^{2}=0$.

for interference cancellation is two when $N^{r x}=7$, whereas $L^{*}=1$ when $N^{r x}=5$.

In Fig. 3, the optimal trade-off between signal power enhancement and interference cancellation for maximizing the spectral efficiency of a typical user in a $12 \times 6 \mathrm{MIMO}$ system is numerically found for various number of users served per resource block and different path-loss exponents. It can be seen that $L^{*}$ moves away from zero with lower number of users served in a resource block and higher pathloss exponents (and vice versa). Under severe propagation conditions, it is therefore best to use all or most antennas to improve the intended signal power. Moreover, in Fig. 4, the optimal number of nearby sources for interference cancellation is plotted against different network deployment densities and SNR operating regions. The figure highlights that for dense network deployments or/and low relative noise power, the optimal number of receive antennas to be used for cancelling interfering streams increases. The trends on the optimal tradeoff between signal power enhancement and interference cancellation can be summarized as: $L^{*}$ increases by having more transmit and receive antennas, fewer users served per resource block, denser deployments, higher SNRs, and larger path-loss exponents (and vice versa).

The area spectral efficiency of full-SDMA cellular systems with different number of transmit antennas is plotted against a wide range of SNR values in Fig. 5. The figure illustrates the following performance trend: (i) area spectral efficiency is improved with higher number of transmit antennas given more UEs can be served simultaneously whilst inter-user interference is eliminated through ZF precoding; and (ii) the performance gain in area spectral efficiency from adding more transmit antennas decreases at higher SNRs. For example, from Fig. 5, the area spectral efficiency of the $8 \times 1$ MIMO system is almost six times higher than that of the baseline

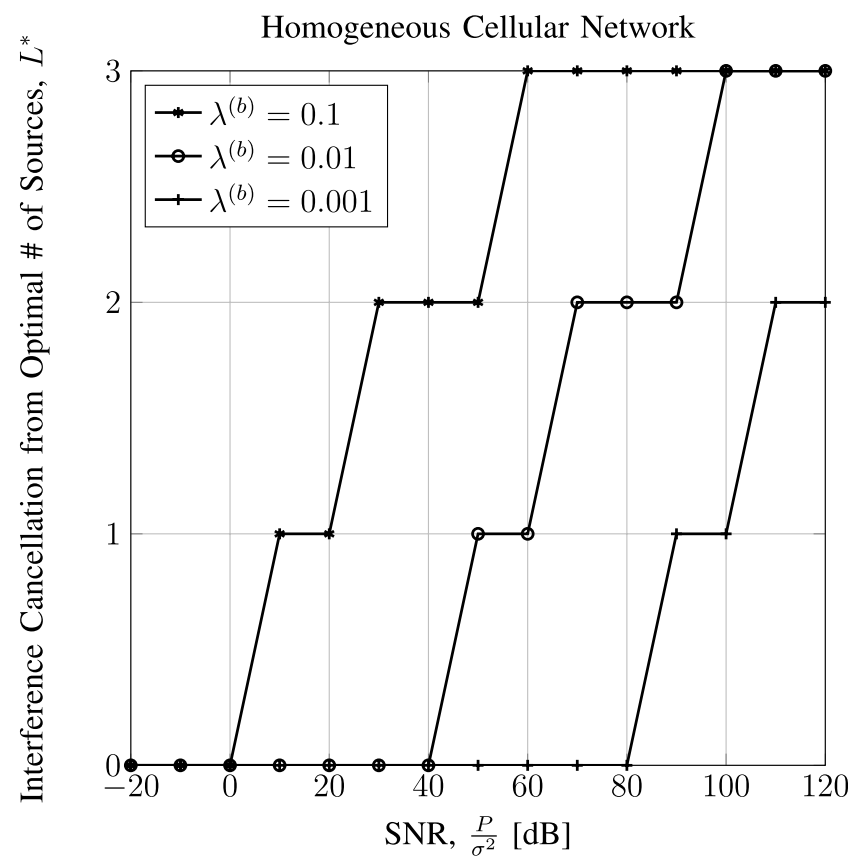

Fig. 4. System parameters are: $N^{t x}=4, N^{r x}=4, U=1, \beta=8$.

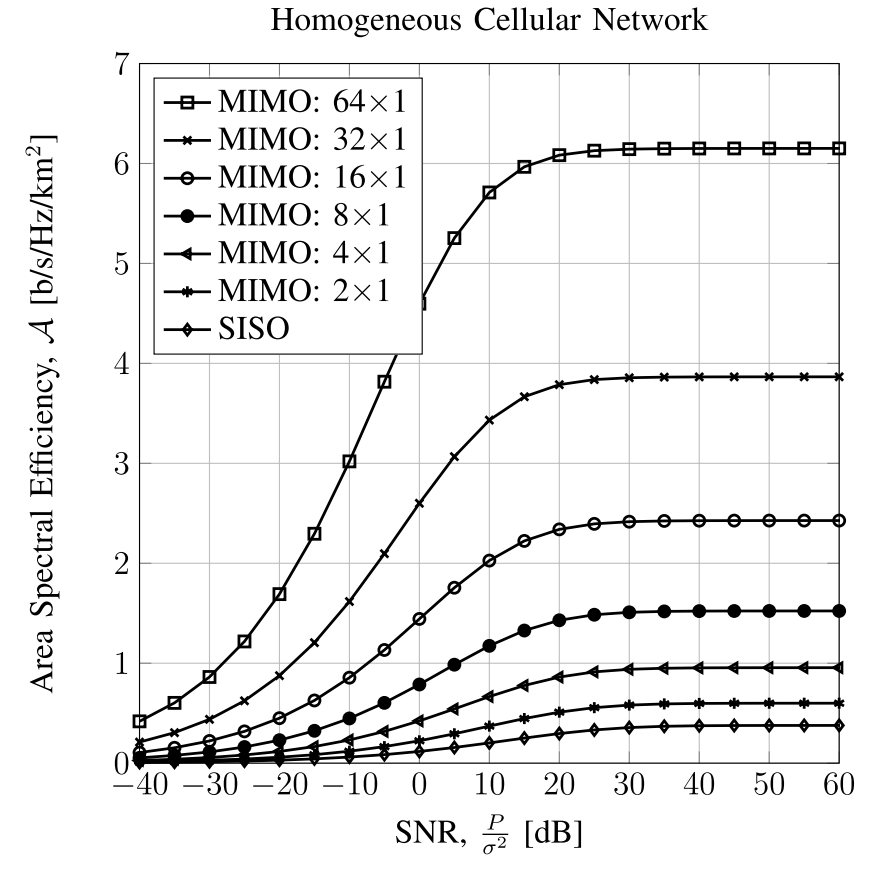

Fig. 5. System parameters are: $\lambda^{(b)}=0.1, U=N^{t x}, L=0, \beta=6$.

SISO system at SNR $=0 \mathrm{~dB}$, whilst the gain is reduced to a near three-fold improvement at $\mathrm{SNR}=40 \mathrm{~dB}$. To gain a better understanding on the impact of different number of antennas at the transmitter and the receiver, we plot the area spectral efficiency with SUBF transmission for a wide range of antenna configurations in Fig. 6. The following network design pointers can be inferred here: (i) the multiplexing gain from adding more transmit antennas results in an almost linear increase in the transmission rate, although the rate of improvement decreases as $N^{t x}$ moves away from one; (ii) the improvement from increasing the number of transmit antennas 
Homogeneous Cellular Network

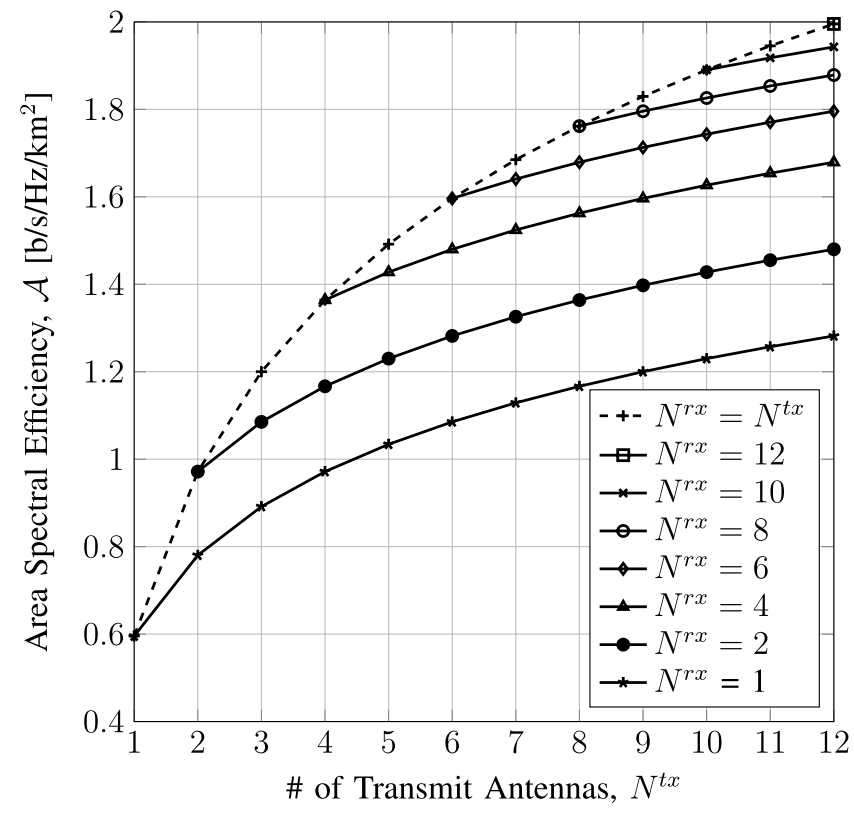

Fig. 6. System parameters are: $\lambda^{(b)}=0.2, U=1, L=0, \mathrm{SNR}=110 \mathrm{~dB}$, $\beta=5$.

is more significant with a lower number of receive antennas used towards full-diversity; and (iii) area spectral efficiency increases when users are equipped with more receive antennas, the improvement however becomes less significant as $N^{r x}$ moves away from one and when more transmit antennas are available.

\section{Multi-Tier Cellular Networks}

We now turn our attention to examining the performance of multi-antenna communications in the stochastic geometrybased HetNet paradigm. Without loss of generality, throughout this section, we consider a two-tier cellular network overlaid with PPP-based macro- and small-cells which differ in terms of their operating parameters. We aim to reflect on the practical aspects associated with HetNet deployment and planning in our choice of system settings. In general, the macro-cells, in comparison to the small-cells, are assumed to be equipped with more transmit antennas and higher total transmit power, and thus capable of serving more UEs in each resource block. In turn, the deployment density and artificial-biasing weights of small-cells are assumed to be generally greater than those of the macro-cells.

The impact of different deployment densities for $8 \times 1$ MIMO macro-cells and $2 \times 1$ small-cells on spectral efficiency of a typical user in the HetNet paradigm is illustrated in Fig. 7. It can be seen from the figure that network densification with small-cells has greatly increased the rate performance of the reference user. The improvement is particularly evident for cases with sparse macro-cell deployments. For example, the spectral efficiency of a typical user in different macro-only networks with densities $\lambda_{m}^{(b)}=0.01$ and $\lambda_{m}^{(b)}=0.005$ is respectively improved by $12.85 \%$ and $29.18 \%$ via overlaying small-cells with a spatial

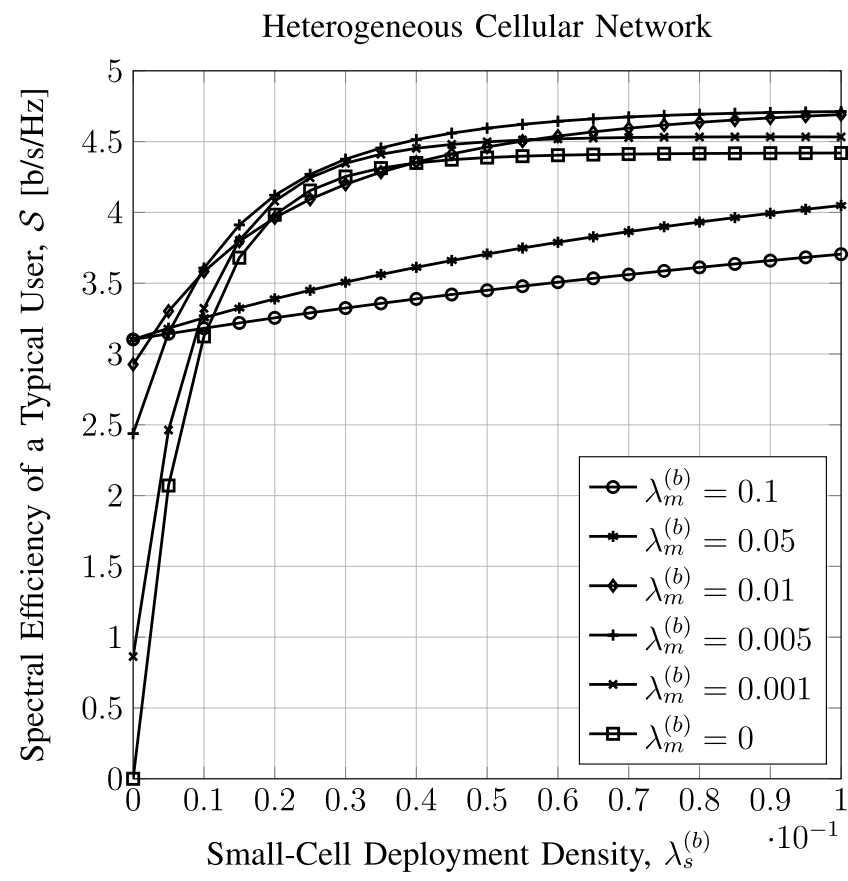

Fig. 7. System parameters are: $N_{m}^{t x}=8, N_{s}^{t x}=2, N^{r x}=1, P_{m}=1$ $\mathrm{W}, P_{s}=0.1 \mathrm{~W}, U_{m}=8, U_{s}=2, L_{m}=L_{s}=0, \varpi_{m}=\varpi_{s}=0 \mathrm{~dB}$, $\frac{1}{\sigma^{2}}=70 \mathrm{~dB}, \beta=8$.

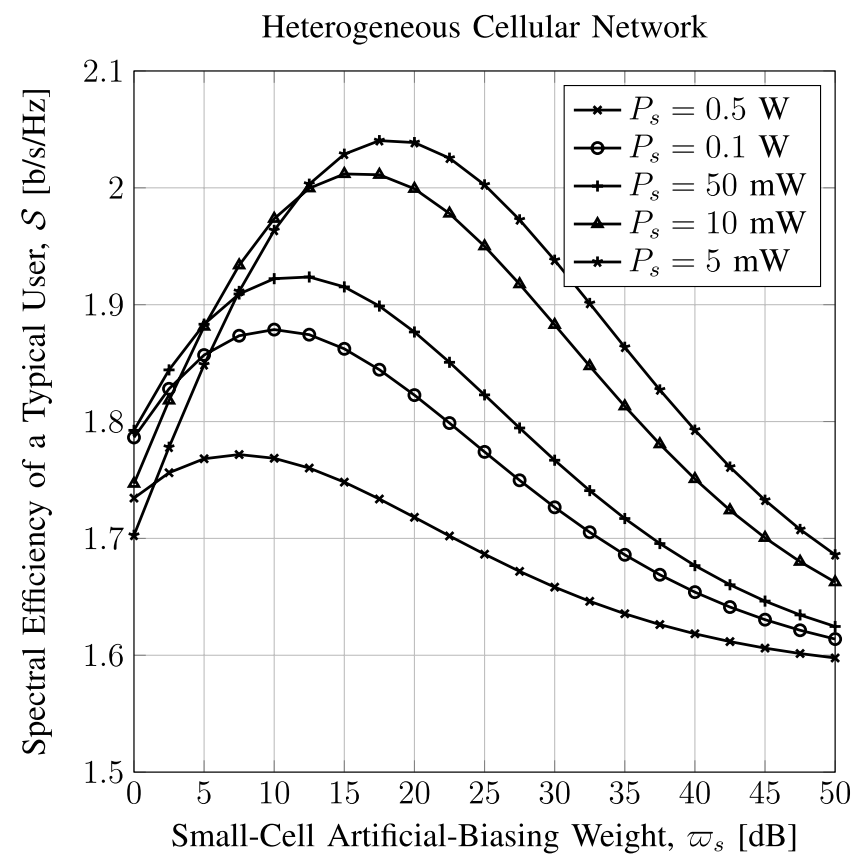

Fig. 8. System parameters are: $\lambda_{m}^{(b)}=0.05, \lambda_{s}^{(b)}=0.25, N_{m}^{t x}=4, N_{s}^{t x}=2$, $N^{r x}=1, P_{m}=2 \mathrm{~W}, U_{m}=4, U_{s}=2, L_{m}=L_{s}=0, \varpi_{m}=0 \mathrm{~dB}, \sigma^{2}=0$, $\beta=4$.

density of $\lambda_{s}^{(b)}=0.05$. As a result of the high inter- and intra-tier interference that deploying macro-cells impose on the system, on the other hand, it can be observed that a sparse macro- and dense small-cell deployment achieves the best user spectral efficiency. For example, consider two macro-only cellular networks with different spatial densities $\lambda_{m}^{(b)}=0.1$ and $\lambda_{m}^{(b)}=0.01$. Without the inclusion of any small-cells, the spectral efficiency experienced by the reference user in the former system is $6.03 \%$ higher than 
Heterogeneous Cellular Network

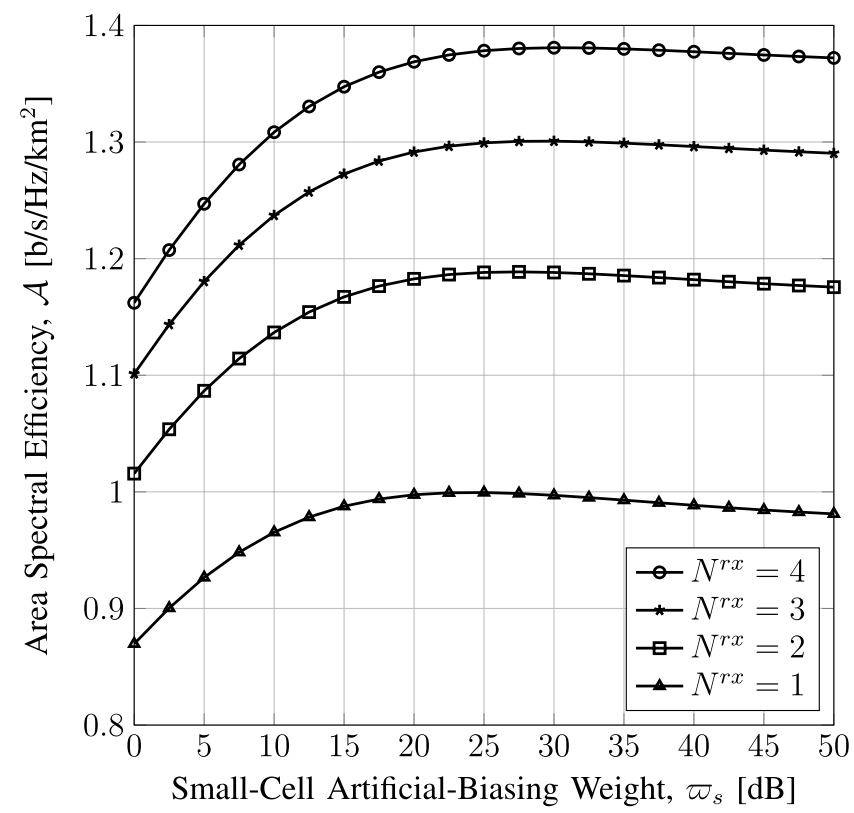

Fig. 9. System parameters are: $\lambda_{m}^{(b)}=0.1, \lambda_{s}^{(b)}=0.2, N_{m}^{t x}=8, N_{s}^{t x}=4$, $P_{m}=1 \mathrm{~W}, P_{s}=0.5 \mathrm{~W}, U_{m}=1, U_{s}=1, L_{m}=L_{s}=0, \varpi_{m}=0 \mathrm{~dB}$, $\sigma^{2}=0, \beta=5$.

that in the latter; however, by overlaying small-cells with a density of $\lambda_{s}^{(b)}=0.1$, the UE in the sparser system achieves a $12.59 \%$ higher $\mathcal{S}$.

Next, we depict the impact of small-cell transmit power and artificial-bias on the spectral efficiency of a typical user in the MIMO HetNet paradigm in Fig. 8. Several important trends can be extracted here. In cases without artificial-bias, it can be observed that selecting an appropriately high transmit power for small-cells improves $\mathcal{S}$. The performance with low transmit powers at the small-cells can particularly be poor due to the limited range and high inter-tier interference from high-power macro-cells. On the other hand, increasing $P_{s}$ beyond a certain level constitutes to higher interference without a significant improvement in the useful signal power. For example, from Fig. 8, with no artificial-bias, with $P_{S}=50 \mathrm{~mW}$, percentage gains of $5.27 \%$ and $3.34 \%$ in $\mathcal{S}$ can be achieved over when $P_{s}=5 \mathrm{~mW}$ and $P_{s}=0.5 \mathrm{~W}$, respectively. Furthermore, it can be observed that with appropriate expansion of small-cells coverage range, performance can be improved. This 'offloading' of traffic from macro-cells to small-cells allows for better overall performance through shortening the transmitterreceiver distance without the penalty of higher interference from increasing $P_{s}$. The optimal biasing choice $\varpi_{s}^{*}$, however, depends on the specific settings of the BSs of different tiers. In general, $\varpi_{s}^{*}$ tends to be greater for lower $P_{s}$ (and vice versa). For instance, from Fig. 8, the best biasing weights when $P_{s}=0.1 \mathrm{~W}$ and $P_{s}=10 \mathrm{~mW}$ are around $10 \mathrm{~dB}$ and $15 \mathrm{~dB}$, respectively. The study of optimal artificial-bias under generalized settings of system parameters is beyond the scope of this paper and is left for future work.

The impact of equipping the UEs with different number of receive antennas on the area spectral efficiency is illustrated

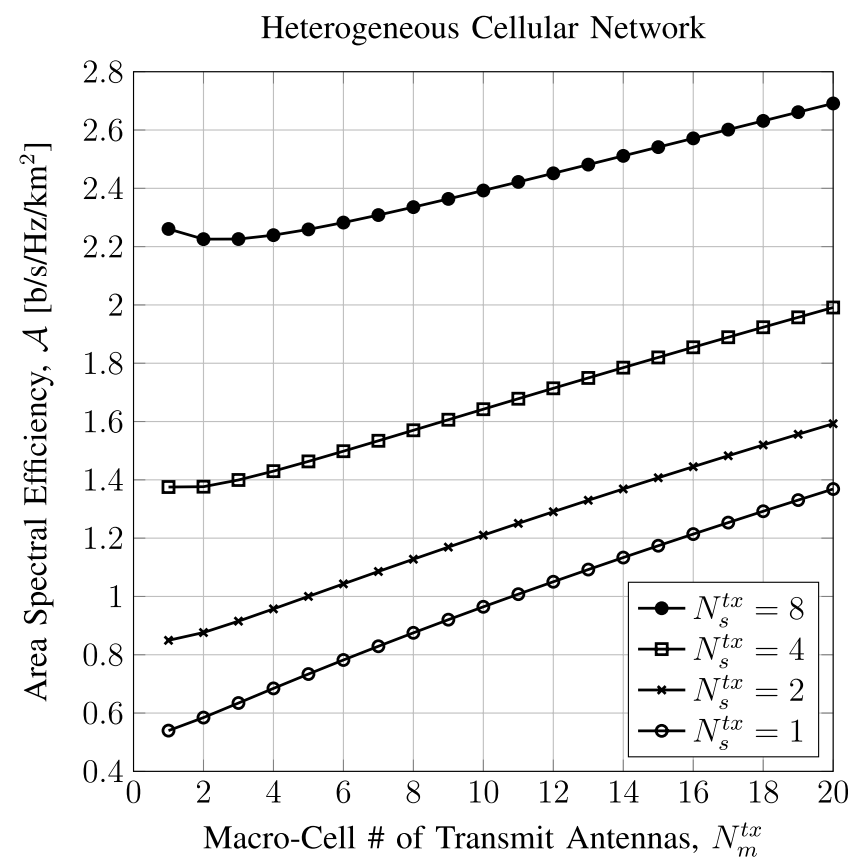

Fig. 10. System parameters are: $\lambda_{m}^{(b)}=0.05, \lambda_{s}^{(b)}=0.2, N^{r x}=1$, $P_{m}=2 \mathrm{~W}, P_{s}=0.2 \mathrm{~W}, U_{m}=N_{m}^{t x}, U_{s}=N_{s}^{t x}, L_{m}=L_{s}=0$, $\varpi_{m}=\varpi_{s}=0 \mathrm{~dB}, \frac{1}{\sigma^{2}}=130 \mathrm{~dB}, \beta=6$.

in Fig. 9. It is observed that by adding more receive antennas, higher values of $\mathcal{A}$ can be realized through utilizing the additional number of antennas towards boosting the intended signal strength. The rate of improvement in HetNet area spectral efficiency, however, diminishes as $N^{r x}$ moves away from one due to the non-linear relative improvement in the useful signal power over aggregate network interference. For example, from Fig. 9, without artificial-bias, the achievable percentage gains in performance from increasing $N^{r x}$ from one to two and three to four are around $16.79 \%$ and $5.53 \%$, respectively. As previously discussed, it can also be observed that the sum-rate can be enhanced via appropriate artificial-biasing weights; further increasing $\varpi_{s}$ however can deteriorate system performance given the low-power small-cells with overly extended reach are not suited to serving distant-located users.

In Fig. 10, we depict the impact of different number of transmit antennas at both macro- and small-cells on the HetNet area spectral efficiency. We can observe that by equipping macro-cells with more transmit antennas, $\mathcal{A}$ increases linearly. It should be noted that the improvement is higher for cases in which the small-cells accommodate fewer users due to having fewer transmit antennas. E.g., increasing $N_{m}^{t x}$ from five to ten results in area spectral efficiency gains of $21.00 \%$ and $12.23 \%$ when $N_{s}^{t x}=2$ and $N_{s}^{t x}=4$, respectively. A similar trend can be observed for the impact of the number of transmit antennas at the small-cells from Fig. 10. It should however be noted that in cases where the number of transmit antennas at low-power small-cells is relatively high, the addition of high-power macro-cells with few transmit antennas amplifies the inter-tier interference resulting in lower area spectral efficiency. 
Heterogeneous Cellular Network

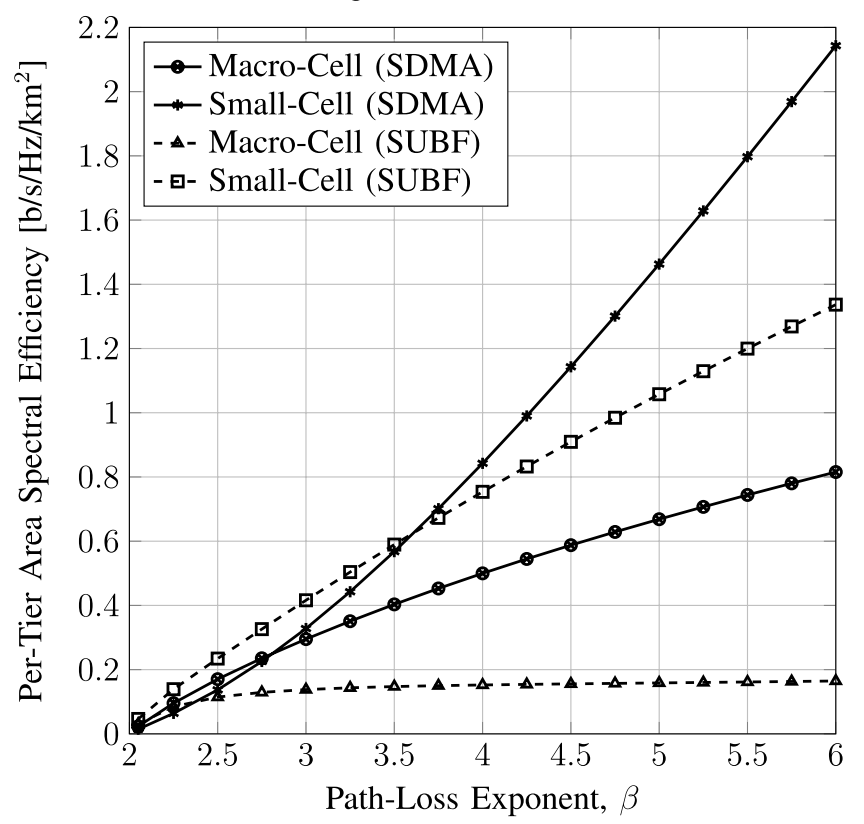

Fig. 11. System parameters are: $\lambda_{m}^{(b)}=0.05, \lambda_{s}^{(b)}=0.35, N_{m}^{t x}=12$, $N_{s}^{t x}=4, N^{r x}=1, P_{m}=2.5 \mathrm{~W}, P_{s}=0.25 \mathrm{~W}, L_{m}=L_{s}=0$, $\varpi_{m}=\varpi_{s}=0 \mathrm{~dB}, \frac{1}{\sigma^{2}}=120 \mathrm{~dB}$.

In Fig. 11 we compare the performance of full-SDMA and SUBF schemes under different path-loss exponents in the MIMO HetNet paradigm. To gain a clear understanding of the performance trends, the attainable area spectral efficiency of each tier is accordingly illustrated. It can be observed that, apart from the case of SDMA under severe path-loss, the small-cell tier outperforms the macro-cell tier irrespective of the transmission scheme employed. This is because the UEs served by the more populated low-power small-cell tier benefit from an overall shorter distance to their BSs. In turn, the high transmission power of macro-cells has negligible impact on performance in medium/high SNR operating regimes. The gain in performance of small- vs macro-cells increases significantly with smaller attenuation from distance-dependent path-loss. For example, from Fig. 11, with SUBF, the small-cell tier provides area spectral efficiency gains of $52.65 \%$ and $201.74 \%$ over the macro-cell tier when $\beta=2.05$ and $\beta=3$, respectively. On the other hand, the graph highlights an important trend where the area spectral efficiency of full-SDMA is superior over SUBF for medium/high values of $\beta$. This is because the added transmit diversity gain from serving one user per resource block is only beneficial over serving as many users as possible when the attenuation of the useful received signal due to the severe propagation conditions is very high. For instance, from Fig. 11, it can be seen that the percentage gains in macro-cell tier area spectral efficiency of SDMA over SUBF are $-22.81 \%$ and $114.00 \%$ when $\beta=2.05$ and $\beta=3$, respectively.

Finally, in Fig. 12, we compare the area spectral efficiency performance of single- and multi-tier cellular networks with different baseline SISO and multi-antenna SDMA transmission

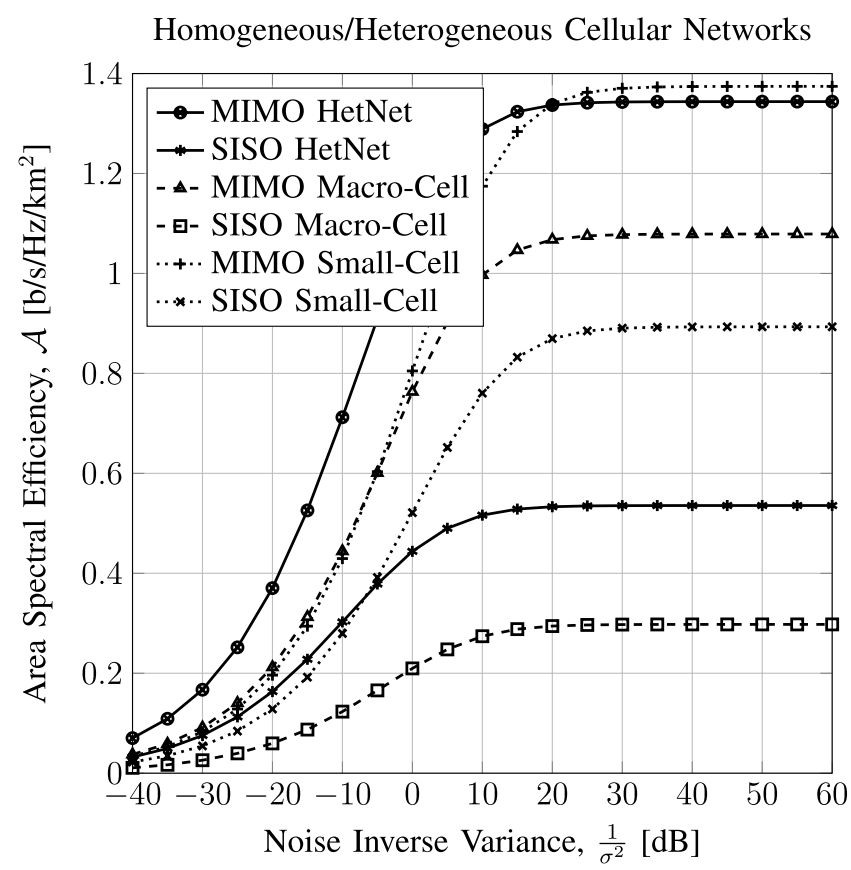

Fig. 12. System parameters are: $\lambda_{m}^{(b)}=\{0.1,0\}, \lambda_{s}^{(b)}=\{0.3,0\}$, $N_{m}^{t x}=\{8,1\}, N_{s}^{t x}=\{2,1\}, N^{r x}=1, N_{m}^{t x} P_{m}=20 \mathrm{~W}, N_{s}^{t x} P_{s}=0.5 \mathrm{~W}$, $U_{m}=N_{m}^{t x}, U_{s}=N_{s}^{t x}, L_{m}=L_{s}=0, \varpi_{m}=\varpi_{s}=0 \mathrm{~dB}, \beta=5$.

schemes. To facilitate a fair comparison, equal per-BS total transmit power is allocated across different systems. Specifically, $8 \times 1$ and $1 \times 1$ macro-cells with per-antenna transmit powers of $2.5 \mathrm{~W}$ and $20 \mathrm{~W}$, and $2 \times 1$ and $1 \times 1$ small-cells with per-antenna transmit powers of $0.25 \mathrm{~W}$ and $0.5 \mathrm{~W}$ are under consideration, respectively. Several important design guidelines can be depicted from the findings. The SISO macrocell deployment is shown to be inferior among the different systems under most SNR settings; this trend highlights the limitations of the conventional sparse high-power macro-only system. The promising potential of multi-antenna communications is further highlighted given a significant gain in area spectral efficiency is achieved through equipping the macrocells with multiple transmit antennas. The SISO HetNet, comprising macro- and small-cells, also performs poorly particularly in interference-limited cases. Furthermore, among all schemes, the heterogeneous MIMO macro- and smallcell and homogeneous MIMO small-cell deployments have achieved the best performance in low and medium/high SNR regions, respectively. In particular, the results highlight the promising potential of multi-antenna small-cellonly deployment in emerging interference-limited cellular networks.

\section{Conclusions}

This paper utilizes tools from stochastic geometry theory to devise a comprehensive analytical model for the design and analysis of multi-antenna multi-tier cellular networks with linear ZF transmit precoding and receive combining. The analysis is facilitated through a computationallyefficient non-direct MGF-based methodology with closed- 
form expressions of the intended signal power and aggregate network interference conditional statistics over Rayleigh fading channels accordingly derived. Extensive Monte-Carlo simulations have verified the validity of the proposed theoretical framework. The findings highlighted the promising potential of multi-antenna techniques at both the transmitter and the receiver for achieving higher user and area spectral efficiencies.

\section{REFERENCES}

[1] N. Bhushan et al., "Network densification: The dominant theme for wireless evolution into 5G," IEEE Commun. Mag., vol. 52, no. 2, pp. 82-89, Feb. 2014

[2] A. Ghosh et al., "Heterogeneous cellular networks: From theory to practice," IEEE Commun. Mag., vol. 50, no. 6, pp. 54-64, Jun. 2012.

[3] A. Damnjanovic et al., "A survey on 3GPP heterogeneous networks," IEEE Trans. Wireless Commun., vol. 18, no. 3, pp. 10-21, Jun. 2011.

[4] J. Tang, D. K. C. So, E. Alsusa, K. A. Hamdi, and A. Shojaeifard, "Resource allocation for energy efficiency optimization in heterogeneous networks," IEEE J. Sel. Areas Commun., vol. 33, no. 10, pp. 2104-2117, Oct. 2015

[5] P. Demestichas et al. " $5 \mathrm{G}$ on the horizon: Key challenges for the radioaccess network," IEEE Veh. Technol. Mag., vol. 8, no. 3, pp. 47-53, Sep. 2013.

[6] R. K. Ganti, F. Baccelli, and J. G. Andrews, "Series expansion for interference in wireless networks," IEEE Trans. Inf. Theory, vol. 58, no. 4, pp. 2194-2205, Apr. 2012.

[7] B. Hagerman, "Downlink relative co-channel interference powers in cellular radio systems," in Proc. IEEE 45th Veh. Technol. Conf., Jul. 1995, pp. 366-370.

[8] H. Tabassum, Z. Dawy, E. Hossain, and M.-S. Alouini, "Interference statistics and capacity analysis for uplink transmission in two-tier small cell networks: A geometric probability approach," IEEE Trans. Wireless Commun., vol. 13, no. 7, pp. 3837-3852, Jul. 2014.

[9] J. G. Andrews, F. Baccelli, and R. K. Ganti, "A tractable approach to coverage and rate in cellular networks," IEEE Trans. Commun., vol. 59, no. 11, pp. 3122-3134, Nov. 2011.

[10] J. Tang, D. K. C. So, E. A. Alsusa, K. Hamdi, and A. Shojaeifard, "On the energy efficiency-spectral efficiency trade-off in MIMO-OFDMA broadcast channels," IEEE Trans. Veh. Technol., to be published.

[11] J. G. Andrews, "Seven ways that HetNets are a cellular paradigm shift," IEEE Commun. Mag., vol. 51, no. 3, pp. 136-144, Mar. 2013.

[12] K. Huang, J. G. Andrews, D. Guo, R. W. Heath, Jr., and R. A. Berry, "Spatial interference cancellation for multiantenna mobile ad hoc networks," IEEE Trans. Inf. Theory, vol. 58, no. 3, pp. 1660-1676, Mar. 2012.

[13] J. G. Andrews, W. Choi, and R. W. Heath, Jr., "Overcoming interference in spatial multiplexing MIMO cellular networks," IEEE Trans. Wireless Commun., vol. 14, no. 6, pp. 95-104, Dec. 2007.

[14] M. Yavuz et al., "Interference management and performance analysis of UMTS/HSPA+ femtocells," IEEE Commun. Mag., vol. 47, no. 9, pp. 102-109, Sep. 2009.

[15] D. Gesbert, S. Hanly, H. Huang, S. Shamai (Shitz), O. Simeone, and W. Yu, "Multi-cell MIMO cooperative networks: A new look at interference," IEEE J. Sel. Areas Commun., vol. 28, no. 9, pp. 1380-1408, Dec. 2010.

[16] J. Tang, D. K. C. So, E. Alsusa, K. A. Hamdi, and A. Shojaeifard, "Energy efficiency optimization with interference alignment in multi-cell MIMO interfering broadcast channels," IEEE Trans. Commun., vol. 63, no. 7, pp. 2486-2499, Jul. 2015.

[17] J. G. Andrews, "Interference cancellation for cellular systems: A contemporary overview," IEEE Trans. Wireless Commun., vol. 12, no. 2, pp. 19-29, Apr. 2005

[18] Q. Zhang, S. Jin, K.-K. Wong, H. Zhu, and M. Matthaiou, "Power scaling of uplink massive MIMO systems with arbitrary-rank channel means," IEEE J. Sel. Topics Signal Process., vol. 8, no. 5, pp. 966-981, Oct. 2014.

[19] N. Jindal, J. G. Andrews, and S. Weber, "Multi-antenna communication in ad hoc networks: Achieving MIMO gains with SIMO transmission," IEEE Trans. Commun., vol. 59, no. 2, pp. 529-540, Feb. 2011.
[20] A. Shojaeifard, K. A. Hamdi, E. Alsusa, D. K. C. So, and J. Tang, "A unified model for the design and analysis of spatially-correlated load-aware HetNets," IEEE Trans. Commun., vol. 62, no. 11, pp. 1-16, Nov. 2014.

[21] H. S. Dhillon, R. K. Ganti, F. Baccelli, and J. G. Andrews, "Modeling and analysis of $\mathrm{K}$-tier downlink heterogeneous cellular networks," IEEE J. Sel. Areas Commun., vol. 30, no. 3, pp. 550-560, Apr. 2012.

[22] G. Alfano, M. Garetto, and E. Leonardi, "New directions into the stochastic geometry analysis of dense CSMA networks," IEEE Trans. Mobile Comput., vol. 13, no. 2, pp. 324-336, Feb. 2014.

[23] H. S. Dhillon, M. Kountouris, and J. G. Andrews, "Downlink MIMO HetNets: Modeling, ordering results and performance analysis," IEEE Trans. Wireless Commun., vol. 12, no. 10, pp. 5208-5222, Oct. 2013.

[24] C. Li, J. Zhang, J. G. Andrews, and K. B. Letaief, "Success probability and area spectral efficiency in multiuser MIMO HetNets," IEEE Trans. Commun., vol. 64, no. 4, pp. 1544-1556, Apr. 2016.

[25] R. W. Heath, Jr., M. Kountouris, and T. Bai, "Modeling heterogeneous network interference using Poisson point processes," IEEE Trans. Signal Process., vol. 61, no. 16, pp. 4114-4126, Aug. 2013.

[26] S. T. Veetil, K. Kuchi, and R. K. Ganti, "Performance of PZF and MMSE receivers in cellular networks with multi-user spatial multiplexing," IEEE Trans. Wireless Commun., vol. 14, no. 9, pp. 4867-4878, Sep. 2015

[27] M. Di Renzo and W. Lu, "Stochastic geometry modeling and performance evaluation of MIMO cellular networks using the equivalentin-distribution (EiD)-based approach," IEEE Trans. Commun., vol. 63, no. 3, pp. 977-996, Mar. 2015.

[28] M. Di Renzo and P. Guan, "Stochastic geometry modeling of coverage and rate of cellular networks using the Gil-Pelaez inversion theorem," IEEE Commun. Lett., vol. 18, no. 9, pp. 1575-1578, Sep. 2014.

[29] D. Stoyan, W. S. Kendall, and J. Mecke, Stochastic Geometry and Its Applications (Wiley Series in Probability and Mathematical Statisitics). New York, NY, USA: Wiley, 1987.

[30] M. Haenggi, "On distances in uniformly random networks," IEEE Trans. Inf. Theory, vol. 51, no. 10, pp. 3584-3586, Oct. 2005.

[31] J. Lee and N. Jindal, "High SNR analysis for MIMO broadcast channels: Dirty paper coding versus linear precoding," IEEE Trans. Inf. Theory, vol. 53, no. 12, pp. 4787-4792, Dec. 2007.

[32] E. Björnson, M. Kountouris, M. Bengtsson, and B. Ottersten, "Receive combining vs. multi-stream multiplexing in downlink systems with multi-antenna users," IEEE Trans. Signal Process., vol. 61, no. 13, pp. 3431-3446, Jul. 2013.

[33] N. Jindal, "Antenna combining for the MIMO downlink channel," IEEE Trans. Wireless Commun., vol. 7, no. 10, pp. 3834-3844, Oct. 2008.

[34] M. Trivellato, F. Boccardi, and H. Huang, "On transceiver design and channel quantization for downlink multiuser MIMO systems with limited feedback," IEEE J. Sel. Areas Commun., vol. 26, no. 8, pp. 1494-1504, Oct. 2008.

[35] Z. Shen, R. Chen, J. G. Andrews, R. W. Heath, Jr., and B. L. Evans, "Low complexity user selection algorithms for multiuser MIMO systems with block diagonalization," IEEE Trans. Signal Process., vol. 54, no. 9, pp. 3658-3663, Sep. 2006.

[36] K. A. Hamdi, "A useful lemma for capacity analysis of fading interference channels," IEEE Trans. Commun., vol. 58, no. 2, pp. 411-416, Feb. 2010.

[37] A. Shojaeifard, K. A. Hamdi, E. Alsusa, D. K. C. So, and J. Tang, "Exact SINR statistics in the presence of heterogeneous interferers," IEEE Trans. Inf. Theory, vol. 61, no. 12, pp. 6759-6773, Dec. 2015.

[38] A. K. Gupta, H. S. Dhillon, S. Vishwanath, and J. G. Andrews, "Downlink coverage probability in MIMO HetNets with flexible cell selection," in Proc. IEEE Global Commun. Conf., Dec. 2014, pp. 1534-1539.

[39] S. Singh, H. S. Dhillon, and J. G. Andrews, "Offloading in heterogeneous networks: Modeling, analysis, and design insights," IEEE Trans. Wireless Commun., vol. 12, no. 5, pp. 2484-2497, May 2013.

[40] A. Guo and M. Haenggi, "Spatial stochastic models and metrics for the structure of base stations in cellular networks," IEEE Trans. Wireless Commun., vol. 12, no. 11, pp. 5800-5812, Nov. 2013. 


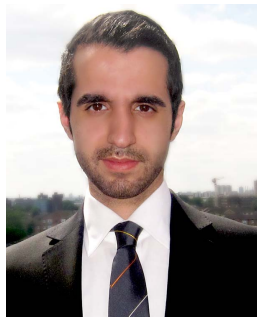

Arman Shojaeifard (S'10-M'13) received the B.Eng. degree in Information Systems Engineering from Imperial College London, U.K., in 2008. He then graduated from King's College London, U.K., in 2009, with the M.Sc. degree in Signal Processing (with Distinction), and in 2013, with the Ph.D degree in Telecommunications. He is currently a Senior Research Associate with the Department of Electronic and Electrical Engineering, University College London, U.K. He has previously held postdoctoral research positions with the Department of Informatics, King's College London, U.K., and the School of Electrical and Electronic Engineering, University of Manchester, U.K. His current research interest is in the area of wireless communications theory, with a particular focus on the design, modeling, performance analysis, and optimization of $5 \mathrm{G}$ cellular networks. He regularly serves as a TPC member for several IEEE flagship conferences including GLOBECOM, ICC, VTC, and PIMRC. He was a recipient of the Exemplary Reviewer Certificate of the IEEE WIRELESS COMMUNICATIONS LETTERS in 2015.

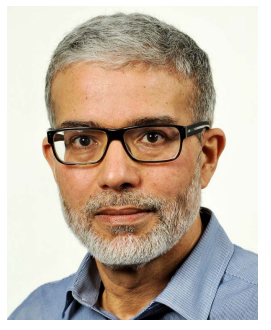

Khairi Ashour Hamdi (M'99-SM'02) received the B.Sc. degree in Electrical Engineering from the University of Tripoli, Libya, in 1981, the M.Sc. degree (with Distinction) from the Technical University of Budapest, Hungary, in 1988, and the Ph.D. degree in Telecommunication Engineering from the Hungarian Academy of Sciences, in 1993. He is now with the School of Electrical and Electronic Engineering, University of Manchester, U.K. Previously, he was with the University of Essex, U.K. His research interests include modeling and performance analysis of wireless communication systems and networks, green communication systems, and heterogeneous mobile networks.

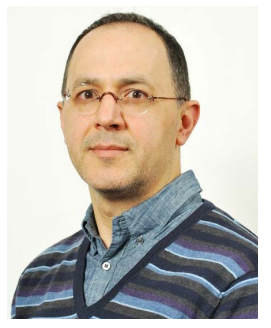

Emad Alsusa (M'06-SM'07) received the Ph.D. degree in Electrical and Electronic Engineering from Bath University, U.K., in 2000. He then joined the School of Engineering and Electronics, Edinburgh University, as a MobileVCE Postdoctoral Research Fellow, working on link enhancement techniques for future high data rate wireless communication systems. In 2003, he became a faculty member at University of Manchester, U.K., where he lectures on communication engineering subjects. His research interests include development of PHY and MAC layers techniques for wired and wireless communication networks, with particular focus on cognitive radio, interference mitigation, multiuser MIMO, spectrum optimization and green systems. He was a co-recipient of the Best Paper Award of the IEEE Symposium on Powerline Communications 2014. He has served as a Technical Program Committee member on numerous IEEE flagship conferences and co-chaired the Greencomm Track in VTC spring 2016.

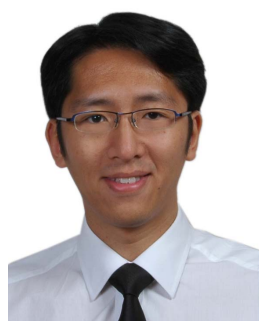

Daniel K. C. So (S'96-M'97-S'98-M'03-SM'14) received the B.Eng. degree in Electrical and Electronic Engineering (First Class Hons.) from the University of Auckland, New Zealand, in 1996, and the Ph.D. Degree in Electrical and Electronic Engineering from the Hong Kong University of Science and Technology, Hong Kong, in 2003. From 1997 to 1998 , he joined the Orion Systems International Ltd., New Zealand, as a Software Engineer, and was later promoted to Senior Software Engineer. Since 2003, he has been a Lecturer and is now a Senior Lecturer in the School of Electrical and Electronic Engineering, University of Manchester, U.K. He has also served as the Director of Postgraduate Taught since 2009. His research interests includes green communications, 5G networks, heterogeneous networks, cognitive radio, non-orthogonal multiple access, massive MIMO, D2D communications, cooperative MIMO schemes, multihop communication, channel equalization and estimation techniques. He is an Editor of IEEE WIRELESS COMMUNICATION LETTERS, and also served as a Track Co-Chair for VTC Spring 2016.

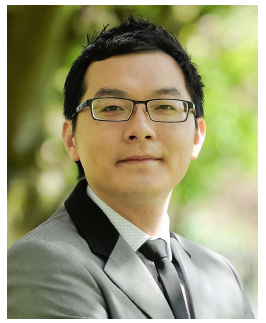

Jie Tang (S'10-M'13) received the B.Eng. degree in Information Engineering from the South China University of Technology, China, in 2008 and the M.Sc. degree (with Distinction) in Communication Systems and Signal Processing from the University of Bristol, UK, in 2009. He received the Ph.D. degree from Loughborough University, U.K., in 2012. From 2013 to 2015, he was a Postdoctoral Research Associate in the School of Electrical and Electronic Engineering, University of Manchester, U.K. Since then, he has been an Associate Professor in the School of Electronic and Information Engineering, South China University of Technology, China. His research interests include optimization techniques and analysis of wireless communication networks, with particular focus on green communications, 5G systems, SWIPT systems, heterogeneous networks, cognitive radio, and MIMO systems.

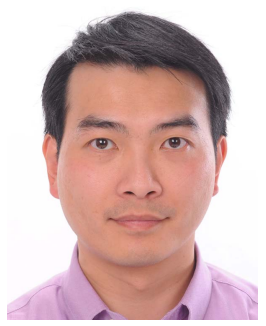

Kai-Kit Wong (M'01-SM'08-F'16) received the B.Eng., M.Phil., and Ph.D. degrees, all in Electrical and Electronic Engineering, from the Hong Kong University of Science and Technology, Hong Kong, in 1996, 1998, and 2001, respectively. He is a Professor of Wireless Communications at the Department of Electronic and Electrical Engineering, University College London, U.K. Prior to this, he held faculty appointments as a Research Assistant Professor at the University of Hong Kong and Lecturer at the University of Hull. He also previously held visiting positions at the Smart Antennas Research Group of Stanford University and the Wireless Communications Research Department of Lucent Technologies, Bell-Labs, Holmdel, NJ, USA Prof. Wong is Fellow of IEEE and IET. $\mathrm{He}$ has been a Senior Editor of the IEEE COMMUNICATIONS LETTERS since 2012 and a Senior Editor of the IEEE WIRELESS COMMUNICATIONS LETTERS since 2016. He has also been on the editorial boards of the IEEE ComSoc/KICS Journal of Communications and Networks (since 2010), IET Communications (since 2009), and Physical Communications (Elsevier; since 2012). He previously served as an Editor of the IEEE TRANSACTIONS ON WiRELESS COMMUNICATIONS from 2005 to 2011, Review Editor of the IEEE COMMUNICATIONS LETTERS from 2009 to 2012, and Associate Editor of the IEEE Signal Processing LetTers from 2009 to 2012. 